\title{
Nonlinear symmetries of perfectly invisible $P T$-regularized conformal and superconformal mechanics systems
}

\author{
Juan Mateos Guilarte ${ }^{a}$ and Mikhail S. Plyushchay ${ }^{b}$ \\ ${ }^{a}$ Departamento de Física Fundamental and IUFFyM, Universidad de Salamanca, \\ Salamanca E-37008, Spain \\ ${ }^{b}$ Departamento de Física, Universidad de Santiago de Chile, \\ Casilla 30\%, Santiago, Chile \\ E-mail: guilarte@usal.es, mikhail.plyushchay@usach.cl
}

ABSTRACT: We investigate how the Lax-Novikov integral in the perfectly invisible PTregularized zero-gap quantum conformal and superconformal mechanics systems affects on their (super)-conformal symmetries. We show that the expansion of the conformal symmetry with this integral results in a nonlinearly extended generalized Shrödinger algebra. The PT-regularized superconformal mechanics systems in the phase of the unbroken exotic nonlinear $\mathcal{N}=4$ super-Poincaré symmetry are described by nonlinearly super-extended Schrödinger algebra with the $\operatorname{osp}(2 \mid 2)$ sub-superalgebra. In the partially broken phase, the scaling dimension of all odd integrals is indefinite, and the $\operatorname{ssp}(2 \mid 2)$ is not contained as a sub-superalgebra.

KEYwords: Conformal and W Symmetry, Extended Supersymmetry, Integrable Hierarchies

ARXIV EPRINT: 1806.08740 


\section{Contents}

1 Introduction and summary 1

$\begin{array}{lll}2 & P T \text {-regularized conformal mechanics models } & 6\end{array}$

3 Symmetries of the $H_{1}^{\alpha}$ system $\quad 7$

4 Symmetries of the $\mathcal{N}=2$ super-extended $H_{1}^{\alpha}$ system 10

5 Symmetries of $H_{n}^{\alpha}$ and of its super-extended version 14

6 Spontaneoulsy broken phase of the exotic SUSY 16

$\begin{array}{llr}7 & \text { Discussion and outlook } & 20\end{array}$

\section{Introduction and summary}

Conformal mechanics model was introduced and investigated by de Alfaro, Fubini and Furlan (AFF) [1] as a (0+1)-dimensional conformal field theory. It corresponds to the two-particle Calogero system [2] with eliminated center of mass degree of freedom. Supersymmetric extension of the model was considered in [3] and [4]. The geometric aspects of conformal and superconformal mechanics were investigated in [5, 6]. The many-particle generalizations of superconformal mechanics were studied in [7-14]. A revival of interest to (super)conformal mechanics was induced in connection with the AdS/CFT correspondence [15-17] when it was observed that the dynamics of a superparticle near the horizon of an extreme Reissner-Nordström black hole is described by superconformal mechanics [18-21]. In the same line of the AdS/CFT correspondence, recently superconformal mechanics was employed in the study of physics underlying the confinement dynamics in QCD [22, 23]. For some further references and reviews on conformal and superconformal mechanics see [24]-[63].

The conformal mechanics model without confining potential term,

$$
H_{g}=-\frac{d^{2}}{d x^{2}}+\frac{g}{x^{2}}, \quad x>0,
$$

is related at special values of the coupling constant $g=n(n+1), n=1,2, \ldots$, to the Korteweg-de Vries (KdV) equation: at $n=1$ its potential is a solution of the stationary $\mathrm{KdV}$ equation, while for $n>1$ it satisfies the $n$-th stationary equation of the KdV hierarchy. This is related to a broader picture according to which the Calogero-Moser systems govern the dynamics of the moving poles of rational solutions to the KdV equation [64-66]. The 
conformal mechanics (1.1) with coupling constant $g=n(n+1)$ plays also a special role in the bispectral problem [67] as well as in the Huygens' principle [68].

The potential of conformal mechanics model with the indicated special values of the coupling constant can be obtained from the potentials of the quantum reflectionless and finite-gap systems via appropriate complex shift of the argument and subsequent application of a certain limit procedure [62]. One-dimensional quantum reflectionless and finite-gap systems, in turn, are the algebro-geometric solutions to equations of the KdV hierarchy via the Lax pair representation [69-71]. Each such quantum system is characterized by a nontrivial Lax-Novikov integral of motion which is a differential operator of odd order $2 n+1 \geq 3$. There exists no classical analog for this integral having a purely quantum origin and nature. It detects all the non-degenerate bound and edge-states by annihilating them, and distinguishes the doubly degenerate states inside the valence and conduction/scattering bands in the spectrum of a corresponding quantum system. In the case of reflectionless systems the integral admits a representation in the form of a Darboux-dressed momentum operator of the free quantum particle [44, 72, 73].

The Lax-Novikov integral also plays an important role in supersymmetric constructions. According to the Burchnall-Chaundy theorem [74, 75], the square of the Lax-Novikov integral of differential order $2 n+1$ is equal to a (spectral) polynomial of the same order $2 n+1$ in Hamiltonian operator of the corresponding quantum system. As a consequence, each non-extended ("purely bosonic") finite-gap or reflectionless quantum mechanical system is characterized by a hidden nonlinear bosonized supersymmetry [72, 73]. On the other hand, it is because of this integral that the convensional $\mathcal{N}=2$ supersymmetry of the pairs of the Darboux-intertwined reflectionless or finite-gap quantum systems expands up to an exotic nonlinear $\mathcal{N}=4$ supersymmetric structure which includes the matrix Lax-Novikov integral as a bosonic central charge [76, 77].

A rather natural question is therefore if the Lax-Novikov integral can be identified for the conformal mechanics model with special values of the coupling constant, and if so, how such integral could influence on the conformal and superconformal symmetries.

Some time ago it was observed that the conformal mechanics model with coupling constant $g=n(n+1)$ possesses a differential operator $P_{n}$ of order $2 n+1$, which commutes with the Hamitlonian $H_{g}$ and satisfies a Burchnall-Chaundy relation of the form $\left(P_{n}\right)^{2}=$ $\left(H_{g}\right)^{2 n+1}[78]$. There appears an obstacle, however, that the operator $P_{n}$ is not physical from the point of view of quantum mechanics : as it was shown in [79], acting on eigenstates of the conformal mechanics model satisfying the Dirichlet boundary condition at $x=0$, it transforms them into formal, non-physical eigenstates of the Hamiltonian operator which satisfy Neumann boundary condition at $x=0$.

In spite of a non-physical nature of the operator $P_{n}$ from the point of view of the quantum mechanical system $H_{g}$ with $g=n(n+1)$, it is the Lax-Novikov operator which underlies the above-mentioned relation of conformal mechanics to the KdV equation and its hierarchy. The Burchnall-Chaundy relation means an algebraic dependence between $H_{g}$ and the formal integral $P_{n}$, and so, the presence of the latter does not influence on integrablity of one-dimensional quantum systems (1.1), which, as any other one-dimensional quantum system with conserved Hamiltonian, is (maximally) super-integrable. However, 
this relation has a super-algebraic nature implying that the Lax-Novikov operator is a kind of a square root operator from the odd order polynomial (monomial here) in Hamiltonian.

In a recent paper [62] it was shown that the indicated deficiency of the Lax-Novikov operator $P_{n}$ can be cured by the $P T$-regularization of the conformal mechanics model by making a shift $x \rightarrow x+i \alpha$, where $\alpha$ is a nonzero real parameter, and extending $x$ from the half-line $x>0$ to the whole real line $x \in \mathbb{R}$. With such a shift and extended domain, the Hamiltonian $H_{n}(x+i \alpha)=-\frac{d^{2}}{d x^{2}}+\frac{n(n+1)}{(x+i \alpha)^{2}}$ is $P T$-symmetric satisfying the relation $\left[P T, H_{n}(x+i \alpha)\right]=0[80,81]$. Here $P$ is a space reflection (parity) operator, $P x=-x P$, $P^{2}=1$, and a complex conjugation operator $T$ is defined by $T z=\bar{z} T, T^{2}=1$, where $z \in \mathbb{C}$ is an arbitrary complex number. ${ }^{1}$ The obtained quantum systems $H_{n}(x+i \alpha)$ are characterized by the property of the perfect invisibility: the transmission amplitude in them is not simply just a phase as it happens in the case of reflectionless systems, but exactly equals one like in the free quantum particle system. Unlike the free quantum particle, however, each such a system has a unique quadratically integrable bound state of zero energy at the very edge of the continuous part of the spectrum. ${ }^{2}$ The corresponding systems are identified as perfectly invisible zero-gap systems, and they possess some other interesting properties due to their relation to the $\mathrm{KdV}$ hierarchy [62]. It may be noted that the parameter $\alpha$ is a constant with dimension of length, and in this aspect the PTregularization of the conformal mechanics model turns out to be alternative in some sense to regularization of the conformal mechanics model considered in the original article [1], where it was realized effectively via the introduction into the Hamiltonian (1.1) of the confining harmonic oscillator potential term accompanied by a length parameter.

It was observed for the first time by Bender and Boettcher in the pioneering work [85] that Hermiticity is not a necessary condition for the reality of the spectrum of a quantum mechanical system, and that it can be substituted for the requirement of the PT-symmetry of Hamiltonian. ${ }^{3}$ Later this type of systems was investigated in different aspects, in particular, in the context of connection between the theories of ordinary differential equations and integrable models [86], and supersymmetry [87]. The results on reality of the spectrum were extended then for non-Hermitian Hamiltonians of a more general form, for reviews see refs. [80] and [81]. The PT-regularization applied in [62] to (1.1) was employed earlier in general context of the $P T$-symmetry for the AFF model with confining potential term [88]. $P T$-symmetric multi-soliton solutions to the Korteweg-de Vries equation were discussed recently in [89], and PT-symmetric deformations of Calogero models were studied in [90, 91]. Nowadays $P T$-symmetry finds interesting applications in diverse areas of physics [92].

- The purpose of this article is to investigate how the Lax-Novikov integral in the $P T$-regularized quantum conformal and superconformal mechanics models affects on their (super)-conformal symmetries.

\footnotetext{
${ }^{1}$ The peculiarity of $P T$-symmetry is associated with the anti-unitary character of the operator $T$ [82].

${ }^{2} \mathrm{~A}$ similar picture appears in finite-gap systems where non-periodic defects can produce bound states at the very edge of the valence and conduction bands [83, 84].

${ }^{3}$ Reality of spectrum in some quantum mechanical systems with non-Hermitian Hamiltonian operators was observed earlier, but the reason of this phenomenon associated with the presence of $P T$-symmetry was established for the first time in [85], see the corresponding discussion in [80].
} 
It is necessary to stress here that the $P T$-regularization $x \rightarrow x+i \alpha$ cannot be considered as a kind of a simple analytic continuation of the models (1.1) since the domain of the latter corresponds to the half-line, while the $P T$-regularized systems are defined on the whole real line $x \in \mathbb{R}$. As we shall see, this results in essential difference in symmetry properties of the $P T$-regularized systems $H_{n}(x+i \alpha)$ and of their supersymmetric versions in comparison with (1.1) and its superextensions. The essential difference can also be expected a priori if, by analogy, we compare the properties of the quantum systems described by potentials $u_{1}=\frac{n(n+1)}{\sinh ^{2} x}$ and $u_{2}=-\frac{n(n+1)}{\cosh ^{2} x}$. Both potentials (shifted for appropriate additive constant terms) are solutions to the corresponding stationary equations of the KdV hierarchy, and the second quantum system is related to the first one by a simple complex shift $x \rightarrow x+i \pi / 2$. The first quantum system, however, is singular at $x=0$ being defined on the real half-line $x>0$ (or $x<0$ ), its non-degenerate spectrum is continuous and has no ground state. The second system is reflectionless being defined on the whole real line, its continuous spectrum is doubly degenerate (except of the non-degenerate state with $E=0$ at the lower edge), and has $n$ non-degenerate negative energies corresponding to bound states, with energy of the ground state $\psi_{0}(x)=1 / \cosh ^{n} x$ equal to $E_{0}=-n^{2}[44,72]$.

Our results can be summarized briefly as follows. We first show that the extension of the set of generators of conformal symmetry of the $P T$-regularized conformal mechanics model with coupling constant $g=2(n=1)$ by its Lax-Novikov integral generates three more dynamical (explicitly depending on time) integrals of motion together with a central charge. As a result, the conformal $s o(2,1) \simeq \operatorname{sl}(2, \mathbb{R})$ Lie algebra expands up to a nonlinearly extended Schrödinger algebra, in which the $s o(2,1)$ generators appear quadratically in commutation relations of the four new non-trivial (including LaxNovikov) integrals of motion. With respect to the adjoint action of the $s o(2,1)$ generators, the complete set of integrals separates into one-dimensional representation corresponding to the mass central charge, while the rest of generators are eigenstates of eigenvalues $(-3 / 2,-1,-1 / 2,0,1 / 2,1,3 / 2)$ of the dilatation generator. The simplest supersymmetric extension of the $P T$-regularized $g=2$ conformal mechanics model is realized via a usual construction of $\mathcal{N}=2$ supersymmetric quantum mechanics based on superpotential $\mathcal{W}_{e}=-1 /(x+i \alpha)$. In this case the $P T$-regularized conformal mechanics model is paired with the free particle, and the system is described by the exotic nonlinear $\mathcal{N}=4$ superPoncare algebra which corresponds to the phase of exact, unbroken supersymmetry with a non-degenerate zero energy ground state. The matrix Lax-Novikov integral is generated by anticommutator of the first and second order supercharges. The extension of the set of generators of the exotic nonlinear $\mathcal{N}=4$ super-Poncaré algebra by the matrix generators of the dilatations and special conformal symmetry transformations gives rise to the nonlinearly extended generalized super-Schrödinger algebra. The set of its nontrivial bosonic generators includes the matrix generalization of the above mentioned seven integrals of motion, the generator of a $u(1) R$-symmetry, and two more integrals which are the momentum and the Galileo boost generator of the free particle subsystem. The set of the fermionic generators includes three pairs of dynamical integrals of motion in addition to the two pairs of the supercharges of the nonlinear $\mathcal{N}=4$ super-Poncaré sub-superalgebra. The superconformal $\operatorname{osp}(2 \mid 2)$ symmetry is the Lie sub-superalgebra, whose expansion by 
any other even or odd integral of motion results in generation of the whole nonlinearly super-extended Schrödinger algebra. All the even and odd integrals form a supermultiplet with respect to the adjoint action of the generators of the $\operatorname{sep}(2 \mid 2)$ superconformal symmetry, and the structure coefficients in the (anti)commutation relations between the rest of the even and odd integrals are linear in generators of the conformal so $(2,1)$ symmetry.

A general case of the $P T$-regularized conformal mechanics with coupling constant $g=$ $n(n+1)$ is characterized by a symmetry described by a nonlinearly extended Schrödinger algebra generated by $2 n+5$ integrals of motion whose scaling dimensions are $(-(n+$ $1 / 2),-(n-1 / 2), \ldots, n-1 / 2, n+1 / 2)$, plus a central charge. The $\mathcal{N}=2$ supersymmetric extension of this $P T$-regularized conformal mechanics model is realized by the construction of supersymmetric quantum mechanical system given by a superpotential $\mathcal{W}_{e}=-n /(x+$ $i \alpha$ ). The obtained in such a way $2 \times 2$ matrix system is characterized by the exotic nonlinear $\mathcal{N}=4$ super-Poincaré type symmetry, in which the anticommutator of the supercharges, being operators of differential orders 1 and $2 n$, generates the matrix Lax-Novikov integral. Extension of this nonlinear $\mathcal{N}=4$ super-Poincaré algebra by generators of dilatations and special conformal transformations results in expansion of the superalgebra up to a nonlinear super-extended Shrödinger symmetry, which contains the superconformal osp (2|2) algebra as a Lie sub-superalgebra. With respect to the adjoint action of the $\operatorname{ssp}(2 \mid 2)$ generators, the rest of the $4 n+2$ bosonic and $4 n+2$ fermionic integrals form an irreducible represenation. The structure coefficients in (anti)-commutation relations between additional even and odd generators are polynomials of order $2 n-1$ in generators of the conformal $s o(2,1)$ symmetry of the system.

We also consider supersymmetric system given by the superpotential $\mathcal{W}_{b}=1 /(x+$ $\left.i \alpha_{1}\right)-1 /\left(x+i \alpha_{2}\right)+i /\left(\alpha_{1}-\alpha_{2}\right), \mathbb{R} \ni \alpha_{j} \neq 0, j=1,2, \alpha_{1} \neq \alpha_{2}$. It represents a matrix system of the Darboux-paired PT-regularized conformal mechanics models with $g=2$ characterized by different values of the shift parameters $\alpha_{1}$ and $\alpha_{2}$. This system is described by the spontaneously partially broken phase of the exotic nonlinear $\mathcal{N}=4$ super-Poncaré symmetry. The essential peculiarity of the system is that all its fermionic generators commute nontrivially with the matrix dilatation generator, neither of them has a definite scaling dimension. The superalgebra of the system represents some nonlinear super-extension of the Schrödinger symmetry which does not include the superconformal $o s p(2 \mid 2)$ symmetry as a sub-superalgebra. Its generators transform nontrivially into those of the system given by superpotential $\mathcal{W}_{e}=-1 /(x+i \alpha)$ in the limit when one of the $P T$-regularization parameters is sent to infinity.

The paper is organized as follows. In section 2, we describe briefly the construction of the perfectly invisible $P T$-regularized zero-gap conformal mechanics systems together with their Lax-Novikov integrals. The nonlinearly extended Schrödinger symmetry of the $P T$-regularized conformal mechanics model with coupling constant $g=2$ is discussed in section 3. Symmetries of the simplest superconformal extension of this system are investigated in section 4 . The results of the two previous sections are generalized for the case of $g=n(n+1)$ in section 5 . The case of the super-extended conformal mechanics with $g=2$ in the phase of spontaneously partially broken exotic nonlinear $\mathcal{N}=4$ super-Poincaré symmetry is considered in section 6 . The concluding discussion is presented in section 7 . 


\section{$2 \quad P T$-regularized conformal mechanics models}

Let us re-denote the Hamiltonian operator of the conformal mechanics model (1.1) with non-negative coupling constant $g=\nu(\nu+1), \nu \geq 0$, as $H_{\nu}$. It admits two factorizations $H_{\nu}=A_{\nu} A_{\nu}^{\#}=A_{\nu+1}^{\#} A_{\nu+1}$ in terms of the first order differential operators $A_{\nu}=x^{\nu} \frac{d}{d x} x^{-\nu}=$ $\frac{d}{d x}-\frac{\nu}{x}, A_{\nu}^{\#}=A_{\nu}^{\dagger}=-\frac{d}{d x}-\frac{\nu}{x}$. The $A_{\nu}$ and $A_{\nu}^{\#}$ intertwine the Hamiltonian operators with different in one values of the index: $A_{\nu} H_{\nu-1}=H_{\nu} A_{\nu}, A_{\nu}^{\#} H_{\nu}=H_{\nu-1} A_{\nu}^{\#}$. The system $H_{\nu}$ is defined on the half-axis $x>0$, and the case with $\nu=0$ can be considered as a limit case $\nu \rightarrow 0$ corresponding to the quantum free particle on the half-line $x>0$ subject to the Dirichlet boundary condition $\psi(0)=0$ [62]. We denote the Hamiltonian operator of such a limit system by $H_{0}^{+}$. The case of integer values of the parameter $\nu=n$ is special in the sense that the conformal mechanics model Hamiltonian $H_{n}$ can be intertwined with that of $H_{0}^{+}, \mathcal{A}_{n}^{\#} H_{n}=H_{0}^{+} \mathcal{A}_{n}^{\#}, \mathcal{A}_{n} H_{0}^{+}=H_{n} \mathcal{A}_{n}$, by the generators of the Darboux-Crum transformation given by the $n$-th order differential operators $\mathcal{A}_{n}=A_{n} A_{n-1} \ldots A_{1}$ and $\mathcal{A}_{n}^{\#}=\mathcal{A}_{n}^{\dagger}$. In correspondence with this, the eigenstates $\psi_{k}^{(0)}(x)=\sin k x$ of $H_{0}^{\dagger}$ are mapped into eigenstates of $H_{n}, \psi_{k}^{(n)}(x)=\mathcal{A}_{n} \psi_{k}^{(0)}(x), H_{n} \psi_{k}^{(n)}=k^{2} \psi_{k}^{(n)}$. Unlike the quantum free particle system $H_{0}=-\frac{d^{2}}{d x^{2}}, x \in \mathbb{R}$, the half-free particle system $H_{0}^{+}$is not translation invariant and $P_{0}=-i \frac{d}{d x}$ is not its physical operator (observable) since acting on the states $\psi_{k}^{(0)}(x)$ it transforms them into the wave functions satisfying the Neumann boundary condition $\psi^{\prime}(0)=0$ instead of the Dirichlet boundary condition. As a result, instead of the algebra of Schrödinger symmetry of the free particle on a whole line given by the Hamiltonian $H_{0}$, the conformal mechanics systems $H_{n}$, including the case $H_{0}^{+}$, are described only by the algebra $s l(2, \mathbb{R})$ of its conformal symmetry subgroup.

By virtue of the intertwining relations between $H_{n}$ and $H_{0}^{+}$, each of the systems $H_{n}$ possesses a formal integral of motion $P_{n}=\mathcal{A}_{n} P_{0} \mathcal{A}_{n}^{\#},\left[P_{n}, H_{n}\right]=0$, which, similarly to the operator $P_{0}$ for $H_{0}^{+}$, 'conflicts' with the Dirichlet boundary condition, and so, is not a physical operator. This 'deficiency' can be removed by the $P T$-symmetric regularization of conformal mechanics systems $H_{n}$ via a purely imaginary shift of the argument, $x \rightarrow x+i \alpha$, $\mathbb{R} \ni \alpha \neq 0$, accompanied by extension of $x$ from the half-line to the whole real line. As it was shown in [62], the resulting Hamiltonian

$$
H_{n}^{\alpha}=-\frac{d^{2}}{d x^{2}}+\frac{n(n+1)}{(x+i \alpha)^{2}}, \quad x \in \mathbb{R}
$$

describes a perfectly invisible zero-gap $P T$-symmetric system, which is characterized by a purely real spectrum in conformity with general properties of such class of the systems $[80,81]$. The peculiarity of the system (2.1), however, is that its transmission amplitude is equal to one for all values of energy $E>0$, and it has one bound state of zero energy given by a square-integrable on $\mathbb{R}$ wave function $\psi_{0}^{(n)}(x)=1 /(x+i \alpha)^{n}$ generated from the eigenstate $\psi_{0}^{(0)}=1$ of zero energy of the free particle system: $\psi_{0}^{(n)}=\mathcal{A}_{n}^{\alpha} \psi_{0}^{(0)}$, where $\mathcal{A}_{n}^{\alpha}=A_{n}^{\alpha} A_{n-1}^{\alpha} \ldots A_{1}^{\alpha}, A_{l}^{\alpha}=\frac{d}{d x}-\frac{l}{x+i \alpha}$. The Lax-Novikov integral

$$
P_{n}^{\alpha}=\mathcal{A}_{n}^{\alpha} P_{0} \mathcal{A}_{n}^{\alpha \#}
$$


where $\mathcal{A}_{n}^{\alpha \#}=A_{1}^{\alpha \#} \ldots A_{n}^{\alpha \#}, A_{l}^{\alpha \#}=-\frac{d}{d x}-\frac{l}{x+i \alpha}$, detects the ground state $\psi_{0}^{(n)}(x)$ by annihilating it, and distinguishes the deformed plane wave eigenstates $\psi^{ \pm k}=\mathcal{A}_{n}^{\alpha} e^{ \pm i k x}$ with $E=k^{2}>0$ in the continuous part of the spectrum, $P_{n}^{\alpha} \psi^{ \pm k}= \pm k^{2 n+1} \psi^{ \pm k}$.

We pass over now to investigation of the effect of the presence of the Lax-Novikov integrals on symmetries of the $P T$-regularized conformal mechanics systems $H_{n}^{\alpha}$ and of their $\mathcal{N}=2$ super-extended versions. To this aim we first consider the case of the simplest system $H_{1}^{\alpha}$, and then we study its supersymmetric extension.

\section{Symmetries of the $H_{1}^{\alpha}$ system}

The generator of Galilean transformations $G_{0}=x-2 t P_{0}=x+2 i t \frac{d}{d x}$ of the free quantum particle $H_{0}=-\frac{d^{2}}{d x^{2}}, x \in \mathbb{R}$, depends explicitly on the time parameter $t$, and satisfies Heisenberg equation of motion of the form $i \frac{d}{d t} G_{0}=i \frac{\partial G_{0}}{\partial t}-\left[H_{0}, G_{0}\right]=0$. In correspondence with this property, $G_{0}$ is identified as a dynamical integral of motion. Two other dynamical integrals of motion of $H_{0}$ are generators of dilatations, $D_{0}=\frac{1}{4}\left\{G_{0}, P_{0}\right\}=-\frac{i}{2}\left(x \frac{d}{d x}+\frac{1}{2}\right)-$ $t H_{0}$, and special conformal transformations, $K_{0}=\left(G_{0}\right)^{2}=x^{2}-8 t D_{0}-4 t^{2} H_{0}$. The integrals $G_{0}, D_{0}$ and $K_{0}$ are not translationally-invariant, $G_{0}(x+\tau)=G_{0}(x)+\tau, \mathcal{D}_{0}(x+\tau)=$ $\mathcal{D}_{0}(x)+\frac{1}{2} \tau P_{0}, K_{0}(x+\tau)=K_{0}(x)+\frac{1}{2} \tau G_{0}(x)+\frac{1}{4} \tau^{2}$. The set of integrals $H_{0}, P_{0}, G_{0}, D_{0}$ and $K_{0}$ and the unit operator $\mathbb{I}$ generate the Schrödinger algebra

$$
\begin{aligned}
& {\left[D_{0}, H_{0}\right]=i H_{0}, \quad\left[D_{0}, K_{0}\right]=-i K_{0}, \quad\left[K_{0}, H_{0}\right]=8 i D_{0},} \\
& {\left[D_{0}, P_{0}\right]=\frac{i}{2} P_{0}, \quad\left[D_{0}, G_{0}\right]=-\frac{i}{2} G_{0},} \\
& {\left[H_{0}, G_{0}\right]=-2 i P_{0}, \quad\left[H_{0}, P_{0}\right]=0,} \\
& {\left[K_{0}, P_{0}\right]=2 i G_{0}, \quad\left[K_{0}, G_{0}\right]=0,} \\
& {\left[G_{0}, P_{0}\right]=i \mathbb{I} \text {. }}
\end{aligned}
$$

This Lie algebra describes symmetry of the free particle. It is the semi-direct sum of the conformal algebra $s l(2, \mathbb{R})$ generated by $H_{0}, D_{0}$ and $K_{0}$, and of the one-dimensional Heisenberg algebra generated by $P_{0}, G_{0}$ and $\mathbb{I}$. The identity operator $\mathbb{I}$ (in the chosen units $\hbar=1, m=1 / 2)$ is the mass central element of the algebra. According to eqs. (3.2), (3.3) and (3.4), the generators of translations, $P_{0}$, and Galileo transformations, $G_{0}$, form a doublet under the adjoint action of the generators $H_{0}, K_{0}$ and $D_{0}$ of the conformal $s l(2, \mathbb{R})$ symmetry. The algebra (3.1)-(3.5) is characterized by the automorphism corresponding to a spatial reflection, $\rho_{1}: P_{0} \rightarrow-P_{0}, G_{0} \rightarrow-G_{0}, H_{0} \rightarrow H_{0}, K_{0} \rightarrow K_{0}, D_{0} \rightarrow D_{0}, \mathbb{I} \rightarrow \mathbb{I}$. It also has another automoprphism

$$
\rho_{2}: H_{0} \rightarrow K_{0}, \quad K_{0} \rightarrow H_{0}, \quad D_{0} \rightarrow-D_{0}, \quad P_{0} \rightarrow-G_{0}, \quad G_{0} \rightarrow P_{0}, \quad \mathbb{I} \rightarrow \mathbb{I},
$$

which at $t=0$ corresponds to a unitary (canonical) transformation $x \rightarrow-i \frac{d}{d x},-i \frac{d}{d x} \rightarrow-x$.

Let us look now for extension and generalization of the Schrödinger Lie algebra (3.1)(3.5) for the case of the PT-regularized conformal mechanics model $H_{1}^{\alpha}$. For this we first identify formally the integrals for the conformal mechanics model $H_{1}$ by Darboux-dressing the integrals of $H_{0}$ and considering their commutation relations. Only generators of the 
conformal $s l(2, \mathbb{R})$ symmetry identified in such a way will be true integrals of motion of $H_{1}$, while other formal integrals will be non-physical: acting on physical eigentstates of $H_{1}$ satisfying the Dirichlet boundary condition $\psi(0)=0$, they produce non-physical states satisfying the Neumann boundary condition $\left(\frac{d}{d x} \psi\right)(0)=0$. The subsequent shift $x \rightarrow x+i \alpha$ (accompanied by extension $x>0 \rightarrow x \in \mathbb{R}$ and omission of boundary condition for wave functions at $x=0$ ) transforms then all the true and formal integrals of $H_{1}$ into the true integrals of motion of the $P T$-regularized conformal mechanics system $H_{1}^{\alpha}$. The key point also is that here the substitution $x \rightarrow x+i \alpha, \frac{d}{d x} \rightarrow \frac{d}{d x}$ changes the operators but does not touch the form of all the corresponding nonlinear algebraic relations they satisfy.

Similarly to a formal (non-physical) integral $P_{1}$ obtained via the Darboux-dressing of the free particle momentum, we identify the analog of $G_{0}$ to be a differential operator

$$
G_{1}(x) \equiv A_{1}(x) G_{0}(x) A_{1}^{\#}(x)=x \frac{d}{d x} A_{1}^{\#}(x)-2 t P_{1}(x) .
$$

Here the argument in integrals $P_{1}(x)$ and $G_{1}(x)$ is shown to stress that they are obtained from the corresponding integrals of the free particle $H_{0}$ via Darboux-dressing by the intertwining operators $A_{1}(x)=\frac{d}{d x}-\frac{1}{x}$ and $A_{1}^{\#}(x)=-\frac{d}{d x}-\frac{1}{x}$. The analog of $D_{0}(x)$ for the system $H_{1}(x)=A_{1}(x) A_{1}^{\#}(x)$ is

$$
D_{1}(x)=-\frac{i}{2}\left(x \frac{d}{d x}+\frac{1}{2}\right)-t H_{1}(x) .
$$

It can be extracted from the Darboux-dressed form of $D_{0}(x)$ by using the relations $A_{1}(x) D_{0}(x) A_{1}^{\#}(x)=\left(D_{1}(x)-\frac{i}{2}\right) H_{1}(x)=H_{1}(x)\left(D_{1}(x)+\frac{i}{2}\right)$, Analogously, from the relations $A_{1}(x) K_{0}(x) A_{1}^{\#}(x)=K_{1}(x) H_{1}(x)-4 i D_{1}(x)-1=H_{1}(x) K_{1}(x)+4 i D_{1}(x)-1$ we extract a dynamical integral

$$
K_{1}(x)=x^{2}-8 t D_{1}(x)-4 t^{2} H_{1}(x) .
$$

The operators $D_{1}, K_{1}$ and $H_{1}$ generate the $s l(2, \mathbb{R})$ algebra of the form (3.1):

$$
\left[D_{1}, H_{1}\right]=i H_{1}, \quad\left[D_{1}, K_{1}\right]=-i K_{1}, \quad\left[K_{1}, H_{1}\right]=8 i D_{1} .
$$

The commutation relations

$$
\left[H_{1}, G_{1}\right]=-2 i P_{1}, \quad\left[H_{1}, P_{1}\right]=0,
$$

are a direct analog of (3.3), while relations (3.2) and the first relation from (3.4) are replaced by

$$
\begin{aligned}
{\left[D_{1}, P_{1}\right] } & =\frac{3}{2} i P_{1}, \quad\left[D_{1}, G_{1}\right]=\frac{i}{2} G_{1}, \\
{\left[K_{1}, P_{1}\right] } & =6 i G_{1} .
\end{aligned}
$$

Instead of (3.5) we have a nonlinear commutation relation

$$
\left[G_{1}, P_{1}\right]=3 i\left(H_{1}\right)^{2}
$$


The zero commutator $\left[K_{0}, G_{0}\right]=0$ is changed for nonzero one,

$$
\left[K_{1}, G_{1}\right]=-4 i V_{1}
$$

where

$$
V_{1}(x)=i x^{2} A_{1}^{\#}(x)-4 t G_{1}(x)-4 t^{2} P_{1}(x)
$$

is identified as a new explicitly depending on time formal integral of motion for the system $H_{1}$. It has the following commutation relations with other integrals:

$$
\begin{aligned}
& {\left[V_{1}, H_{1}\right]=4 i G_{1}, \quad\left[V_{1}, D_{1}\right]=\frac{i}{2} V_{1}, \quad\left[V_{1}, K_{1}\right]=2 i R_{1},} \\
& {\left[V_{1}, P_{1}\right]=12 i H_{1} D_{1}-6 H_{1}, \quad\left[V_{1}, G_{1}\right]=12 i\left(D_{1}\right)^{2}+\frac{3}{4} i \mathbb{I} .}
\end{aligned}
$$

Here the operator

$$
R_{1}(x)=x^{3}-6 t V_{1}(x)-12 t^{2} G_{1}(x)-8 t^{3} P_{1}
$$

has to be identified as yet another new formal dynamical integral of motion of $H_{1}$. Its commutation relations with the rest of the integrals are

$$
\begin{aligned}
& {\left[R_{1}, H_{1}\right]=6 i V_{1}, \quad\left[R_{1}, D_{1}\right]=\frac{3}{2} i R_{1}, \quad\left[R_{1}, K_{1}\right]=0,} \\
& {\left[R_{1}, P_{1}\right]=36 i D_{1}^{2}+\frac{21}{4} i \mathbb{I}, \quad\left[R_{1}, G_{1}\right]=12 i D_{1} K_{1}-6 K_{1}, \quad\left[R_{1}, V_{1}\right]=3 i K_{1}^{2} .}
\end{aligned}
$$

It may be noted here that the commutation relations of the form similar to (3.13) are satisfied by ladder operators in rationally deformed harmonic oscillator systems as well as in rationally deformed conformal mechanics model of de Alfaro-Fubini-Furlan with the included confining harmonic potential term [93, 94].

The explicitly depending on time formal integrals of $H_{1}$ have been identified via the Darboux-dressing of the corresponding integrals of the free particle with additional step of subsequent 'extraction' in the case of the integrals $D_{1}$ and $K_{1}$. The dynamical integrals also can be obtained via the "time-dressing" by the evolution operator $U_{1}(t)=\exp \left(i H_{1} t\right)$. For this we note that the dynamical integral $X_{1}(t)=U_{1}^{-1}(t) x U_{1}(t)$ is given by an infinite series in $t$ and $\frac{d}{d x}$, and so, is a nonlocal in $x$ operator. This is essentially different from the free particle case where $U_{0}(t)=\exp \left(i H_{0} t\right)$ and $U_{0}^{-1}(t) x U_{0}(t)=G_{0}$ is the local operator $G_{0}$. However, the time dressing of the operators $x^{2}, \frac{1}{4}\left\{x,-i \frac{d}{d x}\right\}, x \frac{d}{d x} A_{1}^{\#}, i x^{2} A_{1}^{\#}(x)$ and $x^{3}$ generates the local operators to be exactly the dynamical integrals of motion $K_{1}(x)$, $D_{1}(x), G_{1}(x), V_{1}(x)$ and $R_{1}(x)$, respectively.

The integrals $P_{1}$ and $G_{1}$, being Darboux-dressed free particle's generators of translations, $P_{0}$, and Galileo transformations, $G_{0}$, are the third order differential operators. ${ }^{4}$ According to (3.2) and (3.11), the scaling dimensions $-1 / 2$ and $+1 / 2$ of $P_{0}$ and $G_{0}$ given by relation $i\left[D_{0}, F\right]=s_{F} F$, are changed here for the scaling dimensions $-3 / 2$ and $-1 / 2$ of $P_{1}$ and $G_{1}$ given by analogous relation with $D_{0}$ changed for $D_{1}$. Coherently with this, the central charge in commutation relation (3.5) is changed in (3.13) for the operator $\left(H_{1}\right)^{2}$

\footnotetext{
${ }^{4}$ The not-depending explicitly on $t$ term in (3.7) is the second order differential operator.
} 
having the scaling dimension -2 . In addition, two new formal dynamical integrals $V_{1}$ and $R_{1}$ of the scaling dimensions $+1 / 2$ and $+3 / 2$ are generated via the commutation of $G_{1}$ with generator of the special conformal transformations $K_{1}$ having the scaling dimension +1 . As a result, instead of the Lie algebraic structure of the Schrödinger symmetry of the free particle we obtain the nonlinear algebra in which the commutators (3.13), (3.17) and (3.20) are quadratic in generators of the $s l(2, \mathbb{R})$ Lie subalgebra (3.10). Instead of the doublet of integrals $\left(G_{0}, P_{0}\right)$ under the adjoint action of the operators $H_{0}, K_{0}$ and $D_{0}$ in the case of the free particle, here we have the quartet $\left(R_{1}, V_{1}, G_{1}, P_{1}\right)$ under the adjoint action of the $\operatorname{sl}(2, \mathbb{R})$ generators $H_{1}, K_{1}$ and $D_{1}$.

The described nonlinear algebra is characterized by the automorphism corresponding to a spatial reflection $\rho_{1}$. It also has the automorphism $\rho_{2}$,

$$
\rho_{2}: H_{1} \rightarrow K_{1} \rightarrow H_{1}, D_{1} \rightarrow-D_{1}, P_{1} \rightarrow-R_{1}, R_{1} \rightarrow P_{1}, V_{1} \rightarrow-G_{1}, G_{1} \rightarrow V_{1} .
$$

Till the moment we discussed the integrals of the system $H_{1}$. Except the generators $H_{1}, D_{1}$ and $K_{1}$ of the $s l(2, \mathbb{R})$ symmetry, the rest of them are formal integrals being nonphysical operators. By shifting the argument $x \rightarrow x+i \alpha$ and extending $x>0$ for $x \in \mathbb{R}$, we obtain the corresponding set of the true integrals $H_{1}^{\alpha}, D_{1}^{\alpha}, K_{1}^{\alpha}, P_{1}^{\alpha}, G_{1}^{\alpha}, V_{1}^{\alpha}$ and $R_{1}^{\alpha}$ of the $P T$-regularized conformal mechanics system $H_{1}^{\alpha}$. They satisfy the nonlinearly extended Schrödinger algebra of the same described form.

\section{Symmetries of the $\mathcal{N}=2$ super-extended $H_{1}^{\alpha}$ system}

Consider now the extended system described by the diagonal matrix Hamiltonian operator

$$
\mathcal{H}^{\alpha}=\operatorname{diag}\left(H_{1}^{\alpha}, H_{0}\right)
$$

composed from the $P T$-regularized conformal mechanics Hamiltonian $H_{1}^{\alpha}$ and the free particle Hamiltonian $H_{0}$. To simplify notations, below we omit the upper index $\alpha$ in the Hamiltonian and matrix integrals of the system (4.1). The system (4.1) is described by the superpotential $\mathcal{W}_{e}=-1 /(x+i \alpha), \mathcal{H}=-\frac{d^{2}}{d x^{2}}+\mathcal{W}_{e}^{2}-\mathcal{W}_{e}^{\prime} \sigma_{3}, \mathcal{W}_{e}^{\prime}=\frac{d}{d x} \mathcal{W}_{e}$, and is characterized by the supercharges

$$
\mathcal{Q}_{1}=\left(\begin{array}{cc}
0 & A_{1}^{\alpha} \\
A_{1}^{\alpha \#} & 0
\end{array}\right), \quad \mathcal{Q}_{2}=i \sigma_{3} \mathcal{Q}_{1},
$$

which are the matrix first order differential operators, where $A_{1}^{\alpha}=\frac{d}{d x}+\mathcal{W}_{e}(x), A_{1}^{\alpha \#}=$ $-\frac{d}{d x}+\mathcal{W}_{e}(x)$. The peculiarity of the system (4.1) is that besides the generators of the $\mathcal{N}=2$ supersymmetry $\mathcal{Q}_{a}, a=1,2$, it also possesses two more supercharges

$$
\mathcal{S}_{1}=\left(\begin{array}{cc}
0 & -i A_{1}^{\alpha} P_{0} \\
i P_{0} A_{1}^{\alpha \#} & 0
\end{array}\right), \quad \mathcal{S}_{2}=i \sigma_{3} \mathcal{S}_{1} .
$$

The appearance of additional supercharges (4.3) to be matrix differential operators of the second order, as it clear from their structure, is explained by existence of the momentum 
integral $P_{0}$ in the free particle system. As a consequence, the Hamiltonian operator $H_{1}^{\alpha}$ can be intertwined with the free particle Hamiltonian $H_{0}$ not only by the first order operators $A_{1}^{\alpha}$ and $A_{1}^{\alpha \#}$ but also by the second order operators from which supercharges (4.3) are composed. These four supercharges satisfy the relations

$$
\begin{aligned}
{\left[\mathcal{H}, \mathcal{Q}_{a}\right] } & =0, & {\left[\mathcal{H}, \mathcal{S}_{a}\right]=0, } \\
\left\{\mathcal{Q}_{a}, \mathcal{Q}_{b}\right\} & =2 \delta_{a b} \mathcal{H}, & \left\{\mathcal{S}_{a}, \mathcal{S}_{b}\right\}=2 \delta_{a b} \mathcal{H}^{2}, \\
\left\{\mathcal{Q}_{a}, \mathcal{S}_{b}\right\} & =2 \epsilon_{a b} \mathcal{L}, & \\
{[\mathcal{L}, \mathcal{H}] } & =\left[\mathcal{L}, \mathcal{Q}_{a}\right]=\left[\mathcal{L}, \mathcal{S}_{a}\right]=0, &
\end{aligned}
$$

where

$$
\mathcal{L}=\operatorname{diag}\left(P_{1}^{\alpha}, H_{0} P_{0}\right)
$$

is the bosonic integral of motion composed from the Lax-Novikov integral $P_{1}^{\alpha}$ of the subsystem $H_{1}^{\alpha}$ and the momentum operator $P_{0}$ of the free particle subsystem $H_{0}$. The integrals $\mathcal{H}, \mathcal{Q}_{a}, \mathcal{S}_{a}$ and $\mathcal{L}$ generate the exotic nonlinear $\mathcal{N}=4$ superalgebra of the system (4.1), in which the operator $\mathcal{L}$ plays a role of the bosonic central charge. System (4.1) has the unique bound state of zero energy given by a square-integrable on $\mathbb{R}$ wave function of the form $\Psi^{t}=\left((x+i \alpha)^{-1}, 0\right)^{t}$, which is annihilated by all the superacharges $\mathcal{Q}_{a}$ and $\mathcal{S}_{a}$ as well as by the Lax-Novikov integral $\mathcal{L}$.

We note here that the extension of the $\mathcal{N}=2$ supersymmetry up to the exotic nonlinear $\mathcal{N}=4$ supersymmetry in general case of the quantum reflectionless and finite-gap systems also is based on existence of the two pairs of intertwiners to be differential operators of the even and odd orders. In finite-gap systems, however, no analog of the free particle system with its proper integral of motion $P_{0}$ does appear. In another way the extension can be related to a presentation of the Lax-Novikov integral in such systems in the form of the product of two non-singular operators of the even and odd differential orders. For the detailed discussion of these aspects see refs. [44, 76, 77, 83, 84, 95], where also an important phenomenon of reduction of higher order intertwining operators to intertwining operators of a lower order is discussed. We consider some example of the reduction below in section 6 . Because of such a reduction mechanism, each finite-gap or reflectionless system, including the $P T$-regularized conformal mechanics systems (2.1), is characterized by a corresponding unique irreducible Lax-Novikov integral: reducible intertwining operators generate the same Lax-Novikov integral up to multiplication by a polynomial in the system's Hamiltonian.

The Hamiltonian (4.1) together with the matrix dynamical bosonic integrals $\mathcal{D}=\operatorname{diag}\left(D_{1}^{\alpha}, D_{0}^{\alpha}\right)$ and $\mathcal{K}=\operatorname{diag}\left(K_{1}^{\alpha}, K_{0}^{\alpha}\right)$ generates the conformal algebra $s l(2, \mathbb{R})$,

$$
[\mathcal{D}, \mathcal{H}]=i \mathcal{H}, \quad[\mathcal{D}, \mathcal{K}]=-i \mathcal{K}, \quad[\mathcal{K}, \mathcal{H}]=8 i \mathcal{D},
$$

where we use the notation $D_{0}^{\alpha}=D_{0}(x+i \alpha), K_{0}^{\alpha}=K_{0}(x+i \alpha)$. Extending this set of the bosonic integrals with the supercharge operators $\mathcal{Q}_{a}$ and $\mathcal{S}_{a}$, and taking all the (anti)commutators of these operators and the new integrals generated in this procedure, we obtain a nonlinear superalgebra which corresponds to some nonlinear extension of the 
super-Schrödinger algebra. It is generated by the set of the bosonic integrals $\mathcal{H}, \mathcal{D}, \mathcal{K}, \mathcal{L}$, $\mathcal{G}, \mathcal{V}, \mathcal{R}, \mathcal{P}_{-}, \mathcal{G}_{-}, \Sigma=\sigma_{3}, \mathcal{I}=\operatorname{diag}(1,1)$, and by the fermionic integrals $\mathcal{Q}_{a}, \mathcal{S}_{a}$, and $\lambda_{a}$, $\mu_{a}$ and $\kappa_{a}, a=1,2$, where

$$
\begin{array}{rlr}
\mathcal{G} & =\operatorname{diag}\left(G_{1}^{\alpha}, \frac{1}{2}\left\{G_{0}^{\alpha}, H_{0}\right\}\right), & \\
\mathcal{V} & =i(x+i \alpha)^{2} A_{1}^{\alpha \#} \mathcal{I}-4 t \mathcal{G}-4 t^{2} \mathcal{L} & \\
\mathcal{R} & =(x+i \alpha)^{3} \mathcal{I}-6 t \mathcal{V}-12 t^{2} \mathcal{G}-8 t^{3} \mathcal{L}, & \mathcal{G}_{-}=\frac{1}{2}\left(1-\sigma_{3}\right) \\
\mathcal{P}_{-} & =\frac{1}{2}\left(1-\sigma_{3}\right) P_{0}, & \lambda_{2}=i \sigma_{3} \lambda_{1}, \\
\lambda_{1} & =\left(\begin{array}{cc}
0 & i(x+i \alpha) \\
-i(x+i \alpha) & 0
\end{array}\right)-2 t \mathcal{Q}_{1}, & \mu_{2}=i \sigma_{3} \mu_{1}, \\
\mu_{1} & =\left(\begin{array}{cc}
0 & (x+i \alpha) P_{0} \\
P_{0}(x+i \alpha) & 0
\end{array}\right)-2 t \mathcal{S}_{1}, & \\
\kappa_{1} & =\left(\begin{array}{cc}
0 & (x+i \alpha)^{2} \\
(x+i \alpha)^{2} & 0
\end{array}\right)-4 t \mu_{1}-4 t^{2} \mathcal{S}_{1}, & \kappa_{2}=i \sigma_{3} \kappa_{1} .
\end{array}
$$

The dynamical integrals represent the corresponding time-independent operators "dressed" by the matrix evolution operator $\mathcal{U}(t)=\exp (i \mathcal{H} t): F(t)=\mathcal{U}^{-1}(t) F(0) \mathcal{U}(t)$. Particularly, the dynamical integral $\mathcal{G}$ corresponds to a dressed form of the diagonal matrix operator $g=\operatorname{diag}\left((x+i \alpha) \frac{d}{d x} A_{1}^{\alpha \#},(x+i \alpha) A_{1}^{\left.\alpha \# \frac{d}{d x}\right) .}\right.$

All the integrals are eigenstates of the operator $\mathcal{D}$ in the sense of its adjoint action:

$$
\begin{aligned}
& {[\mathcal{D}, \mathcal{I}]=[\mathcal{D}, \mathcal{D}]=[\mathcal{D}, \Sigma]=\left[\mathcal{D}, \mu_{a}\right]=0,} \\
& {[\mathcal{D}, \mathcal{H}]=i \mathcal{H}, \quad[\mathcal{D}, \mathcal{K}]=-i \mathcal{K},} \\
& {[\mathcal{D}, \mathcal{L}]=\frac{3}{2} i \mathcal{L}, \quad[\mathcal{D}, \mathcal{R}]=-\frac{3}{2} i \mathcal{R},} \\
& {[\mathcal{D}, \mathcal{G}]=\frac{i}{2} \mathcal{G}, \quad[\mathcal{D}, \mathcal{V}]=-\frac{i}{2} \mathcal{V}, \quad\left[\mathcal{D}, \mathcal{P}_{-}\right]=\frac{i}{2} \mathcal{P}_{-}, \quad\left[\mathcal{D}, \mathcal{G}_{-}\right]=-\frac{i}{2} \mathcal{G}_{-},} \\
& {\left[\mathcal{D}, \mathcal{Q}_{a}\right]=\frac{i}{2} \mathcal{Q}_{a}, \quad\left[\mathcal{D}, \mathcal{S}_{a}\right]=i \mathcal{S}_{a}, \quad\left[\mathcal{D}, \lambda_{a}\right]=-\frac{i}{2} \lambda_{a} \quad\left[\mathcal{D}, \kappa_{a}\right]=-i \kappa_{a}}
\end{aligned}
$$

The complete set of other (anti)-commutation relations is :

$$
\begin{array}{rlrl}
{[\mathcal{H}, \mathcal{K}]} & =-8 i \mathcal{D}, & {[\mathcal{H}, \mathcal{D}]} & =-i \mathcal{H}, \\
{[\mathcal{H}, \mathcal{R}]} & =-6 i \mathcal{V}, \quad[\mathcal{H}, \mathcal{V}] & =-4 i \mathcal{G}, \quad[\mathcal{H}, \mathcal{G}]=-2 i \mathcal{L}, \quad[\mathcal{H}, \mathcal{L}]=0, \\
{\left[\mathcal{H}, \mathcal{G}_{-}\right]} & =-2 i \mathcal{P}_{-}, \quad\left[\mathcal{H}, \mathcal{P}_{-}\right] & =0, \\
{\left[\mathcal{H}, \mathcal{Q}_{a}\right]} & =0, & {\left[\mathcal{H}, \mathcal{S}_{a}\right]} & =0, \\
{\left[\mathcal{H}, \kappa_{a}\right]} & =-4 i \mu_{a}, \quad\left[\mathcal{H}, \mu_{a}\right]=-2 i \mathcal{S}_{a}, \quad\left[\mathcal{H}, \lambda_{a}\right]=-2 i \mathcal{Q}_{a}, \\
{[\mathcal{K}, \mathcal{L}]} & =6 i \mathcal{G}, \quad[\mathcal{K}, \mathcal{G}]=4 i \mathcal{V}, \quad[\mathcal{K}, \mathcal{V}]=2 i \mathcal{R}, \quad[\mathcal{K}, \mathcal{R}]=0, \\
{\left[\mathcal{K}, \mathcal{P}_{-}\right]} & =2 i \mathcal{G}_{-}, \quad\left[\mathcal{K}, \mathcal{G}_{-}\right]=0, \\
{\left[\mathcal{K}, \mathcal{Q}_{a}\right]} & =2 i \lambda_{a}, \quad\left[\mathcal{K}, \lambda_{a}\right]=0, \quad\left[\mathcal{K}, \mathcal{S}_{a}\right]=4 i \mu_{a}, \quad\left[\mathcal{K}, \mu_{a}\right]=2 i \kappa_{a}, \quad\left[\mathcal{K}, \kappa_{a}\right]=0,
\end{array}
$$




$$
\begin{aligned}
& {[\mathcal{L}, \mathcal{G}]=-3 i \mathcal{H}^{2}, \quad\left[\mathcal{L}, \mathcal{P}_{-}\right]=0, \quad\left[\mathcal{L}, \mathcal{G}_{-}\right]=-3 i \Pi_{-} \mathcal{H},} \\
& {[\mathcal{L}, \mathcal{V}]=-3\left(1+\Upsilon^{+}\right) \mathcal{H}, \quad[\mathcal{L}, \mathcal{R}]=-i\left(36 \mathcal{D}^{2}+\frac{9}{2} \Sigma+\frac{3}{4} \mathcal{I}\right)} \\
& {\left[\mathcal{L}, \mathcal{Q}_{a}\right]=0, \quad\left[\mathcal{L}, \mathcal{S}_{a}\right]=0,} \\
& {\left[\mathcal{L}, \lambda_{a}\right]=3 i \epsilon_{a b} \mathcal{S}_{b}, \quad\left[\mathcal{L}, \mu_{a}\right]=3 i \mathcal{H} \epsilon_{a b} \mathcal{Q}_{b}, \quad\left[\mathcal{L}, \kappa_{a}\right]=3\left(\Upsilon^{+} \epsilon_{a b}+i \delta_{a b}\right) \mathcal{Q}_{b},} \\
& {[\mathcal{G}, \mathcal{V}]=3 i \mathcal{H} \mathcal{K}-12 \mathcal{D}+\frac{i}{2}(7 \Sigma-\mathcal{I}), \quad[\mathcal{G}, \mathcal{R}]=3\left(1+\Upsilon^{-}\right) \mathcal{K},} \\
& {\left[\mathcal{G}, \mathcal{P}_{-}\right]=i \Pi_{-} \mathcal{H}} \\
& {\left[\mathcal{G}, \mathcal{G}_{-}\right]=-4 i \Pi_{-} \mathcal{D}} \\
& {\left[\mathcal{G}, \mathcal{Q}_{a}\right]=i \epsilon_{a b} \mathcal{S}_{b},} \\
& {\left[\mathcal{G}, \mathcal{S}_{a}\right]=-2 i \mathcal{H} \epsilon_{a b} \mathcal{Q}_{b},} \\
& {\left[\mathcal{G}, \lambda_{a}\right]=-2 i \epsilon_{a b} \mu_{b},} \\
& {\left[\mathcal{G}, \mu_{a}\right]=\frac{1}{2} \Upsilon^{+} \epsilon_{a b} \mathcal{Q}_{b},} \\
& {\left[\mathcal{G}, \kappa_{a}\right]=-\left(2 \Upsilon^{-} \epsilon_{a b}+i \delta_{a b}\right) \lambda_{b}} \\
& {[\mathcal{V}, \mathcal{R}]=-3 i \mathcal{K}^{2}, \quad\left[\mathcal{V}, \mathcal{P}_{-}\right]=4 i \Pi_{-} \mathcal{D}, \quad\left[\mathcal{V}, \mathcal{G}_{-}\right]=-i \Pi_{-} \mathcal{K}} \\
& {\left[\mathcal{V}, \mathcal{Q}_{a}\right]=2 i \epsilon_{a b} \mu_{b}, \quad\left[\mathcal{V}, \mathcal{S}_{a}\right]=-\left(2 \Upsilon^{+} \epsilon_{a b}+i \delta_{a b}\right) \mathcal{Q}_{b},} \\
& {\left[\mathcal{V}, \lambda_{a}\right]=-i \epsilon_{a b} \kappa_{b}, \quad\left[\mathcal{V}, \mu_{a}\right]=\frac{1}{2} \Upsilon^{-} \epsilon_{a b} \lambda_{b}, \quad\left[\mathcal{V}, \kappa_{a}\right]=4 i \mathcal{K} \epsilon_{a b} \lambda_{b},} \\
& {\left[\mathcal{R}, \mathcal{P}_{-}\right]=3 i \Pi_{-} \mathcal{K}, \quad\left[\mathcal{R}, \mathcal{G}_{-}\right]=0,} \\
& {\left[\mathcal{R}, \mathcal{Q}_{a}\right]=-3 i \epsilon_{a b} \kappa_{b}, \quad\left[\mathcal{R}, \mathcal{S}_{a}\right]=3\left(\Upsilon^{-} \epsilon_{a b}+i \delta_{a b}\right) \lambda_{b},} \\
& {\left[\mathcal{R}, \lambda_{a}\right]=0, \quad\left[\mathcal{R}, \mu_{a}\right]=-3 i \mathcal{K} \epsilon_{a b} \lambda_{b}, \quad\left[\mathcal{R}, \kappa_{a}\right]=0,} \\
& {\left[\mathcal{P}_{-}, \mathcal{G}_{-}\right]=-\frac{i}{2}(\mathcal{I}-\Sigma), \quad\left[\mathcal{P}_{-}, \mathcal{Q}_{a}\right]=-i \mathcal{S}_{a}, \quad\left[\mathcal{P}_{-}, \mathcal{S}_{a}\right]=i \mathcal{H} \mathcal{Q}_{a}} \\
& {\left[\mathcal{P}_{-}, \lambda_{a}\right]=-i \mu_{a}} \\
& {\left[\mathcal{P}_{-}, \mu_{a}\right]=\frac{1}{2} \Upsilon^{+} \mathcal{Q}_{a}, \quad\left[\mathcal{P}_{-}, \kappa_{a}\right]=\left(-\frac{1}{2} \Upsilon^{-} \delta_{a b}+i \epsilon_{a b}\right) \lambda_{b},} \\
& {\left[\mathcal{G}_{-}, \mathcal{Q}_{a}\right]=-i \mu_{a}, \quad\left[\mathcal{G}_{-}, \mathcal{S}_{a}\right]=-\mathcal{Q}_{a}+i \mathcal{H} \lambda_{a},} \\
& {\left[\mathcal{G}_{-}, \lambda_{a}\right]=-i \kappa_{a}, \quad\left[\mathcal{G}_{-}, \mu_{a}\right]=-\frac{1}{2} \Upsilon^{-} \lambda_{a}, \quad\left[\mathcal{G}_{-}, \kappa_{a}\right]=i \mathcal{K} \lambda_{a},} \\
& {[\Sigma, \mathcal{B}]=0, \quad \mathcal{B}=\Gamma, \mathcal{H}, \mathcal{L}, \mathcal{D}, \mathcal{K}, \mathcal{G}, \mathcal{V}, \mathcal{R}, \mathcal{P}_{-}, \mathcal{G}_{-},} \\
& {\left[\Sigma, \mathcal{F}_{a}\right]=2 i \epsilon_{a b} \mathcal{F}_{b}, \quad \mathcal{F}_{a}=\mathcal{Q}_{a}, \mathcal{S}_{a}, \lambda_{a}, \mu_{a}, \kappa_{a}} \\
& \left\{\mathcal{Q}_{a}, \mathcal{Q}_{b}\right\}=2 \delta_{a b} \mathcal{H}, \\
& \left\{\mathcal{Q}_{a}, \mathcal{S}_{b}\right\}=2 \epsilon_{a b} \mathcal{L} \\
& \left\{\mathcal{Q}_{a}, \lambda_{b}\right\}=4 \delta_{a b} \mathcal{D}+2 \epsilon_{a b}\left(\mathcal{I}-\frac{1}{2} \Sigma\right), \\
& \left\{\mathcal{Q}_{a}, \mu_{b}\right\}=-2 \delta_{a b} \mathcal{P}_{-}+2 \epsilon_{a b} \mathcal{G}, \quad\left\{\mathcal{Q}_{a}, \kappa_{b}\right\}=-4 \delta_{a b} \mathcal{G}_{-}+2 \epsilon_{a b} \mathcal{V},
\end{aligned}
$$




$$
\begin{array}{ll}
\left\{\mathcal{S}_{a}, \mathcal{S}_{b}\right\} & =2 \delta_{a b} \mathcal{H}^{2}, \\
\left\{\mathcal{S}_{a}, \lambda_{b}\right\} & =4 \delta_{a b} \mathcal{P}_{-}-2 \epsilon_{a b} \mathcal{G}, \quad\left\{\mathcal{S}_{a}, \mu_{b}\right\}=\left(-i\left(1+\Upsilon^{+}\right) \delta_{a b}+(1-2 \Sigma) \epsilon_{a b}\right) \mathcal{H}, \\
\left\{\mathcal{S}_{a}, \kappa_{b}\right\} & =\left(\frac{1}{2} \mathcal{H} \mathcal{K}-\mathcal{I}\right) \delta_{a b}+\left(2 i \delta_{a b}+4(1-2 \Sigma) \epsilon_{a b}\right) \mathcal{D}, \\
\left\{\lambda_{a}, \lambda_{b}\right\} & =2 \delta_{a b} \mathcal{K}, \quad\left\{\lambda_{a}, \mu_{b}\right\}=2 \epsilon_{a b} \mathcal{V}+2 \delta_{a b} \mathcal{G}_{-}, \quad\left\{\lambda_{a}, \kappa_{b}\right\}=2 \epsilon_{a b} \mathcal{R}, \\
\left\{\mu_{a}, \mu_{b}\right\} & =\frac{1}{2} \Upsilon^{+} \Upsilon^{-} \delta_{a b}, \quad\left\{\mu_{a}, \kappa_{b}\right\}=\left(i\left(1+\Upsilon^{-}\right) \delta_{a b}+(1-2 \Sigma) \epsilon_{a b}\right) \mathcal{K}, \\
\left\{\kappa_{a}, \kappa_{b}\right\} & =2 \delta_{a b} \mathcal{K}^{2} .
\end{array}
$$

We use here a notation $\Pi_{-}=\frac{1}{2}(1-\Sigma)$ and $\Upsilon^{ \pm}=1 \pm 4 i \mathcal{D}$ for operator-valued coefficients appearing in some (anti)-commutation relations.

The superalgebra (4.17)-(4.60) represents a nonlinear generalization of the superSchrödinger symmetry with ten even and ten odd generators plus a bosonic central charge $\mathcal{I}$. It is characterized by the quadratic Casimir operator $C_{0}=\{\mathcal{K}, \mathcal{H}\}-8 \mathcal{D}^{2}+2\left(\mathcal{I}-\frac{1}{2} \Sigma\right)^{2}-3 \mathcal{I}^{2}$, which takes zero value $C_{0}=0$ for the system (4.1). The set of operators $\mathcal{H}, \mathcal{K}, \mathcal{D},\left(\mathcal{I}-\frac{1}{2} \Sigma\right)$, $\mathcal{Q}_{a}$ and $\lambda_{a}$ generates the Lie sub-superalgebra $\operatorname{osp}(2 \mid 2)$ of superconformal symmetry of the system. The extension of the indicated set of operators by the Lax integral $\mathcal{L}$ or by any other integral not appearing in the list produces all the rest of the generators and expands the superalgebra $\operatorname{ssp}(2 \mid 2)$ up to the described nonlinear (quadratic) superalgebra. As an example, one such a chain of the (anti)commutation relations can be presented schematically as

$$
\mathcal{L} \stackrel{\mathcal{K}}{\longrightarrow} \mathcal{G} \stackrel{\mathcal{K}}{\longrightarrow} \mathcal{V} \stackrel{\mathcal{K}}{\longrightarrow} \mathcal{R}, \quad \mathcal{L} \stackrel{\lambda_{a}}{\longrightarrow} \mathcal{S}_{a} \stackrel{\lambda_{a}}{\longrightarrow} \mathcal{P}_{-} \stackrel{\lambda_{a}}{\longrightarrow} \mu_{a} \stackrel{\lambda_{a}}{\longrightarrow} \mathcal{G}_{-} \stackrel{\lambda_{a}}{\longrightarrow} \kappa_{a}, \quad \mathcal{G}_{-} \stackrel{\mathcal{P}_{-}}{\longrightarrow} \mathcal{I}, \Sigma,
$$

where $\mathcal{L} \stackrel{\mathcal{K}}{\longrightarrow} \mathcal{G}$ corresponds to the first commutation relation in (4.27), etc.

The peculiarity of the obtained nonlinear superalegbra is that the (anti)-commutators of the generators of the $\operatorname{ssp}(2 \mid 2)$ sub-superalgebra with any other generator is linear in generators; at the same time, in the (anti)-commutators of the rest of the integrals, the generators $\mathcal{H}, \mathcal{K}, \mathcal{D}$ and $\Sigma$ of the $\operatorname{sl}(2, \mathbb{R}) \oplus u(1)$ sub-algebra appear as operator-valued coefficients.

The superalgebra has the automorphism corresponding to a spatial reflection $\rho_{1}$, under which the integrals $\mathcal{L}, \mathcal{G}, \mathcal{V}, \mathcal{R}, \mathcal{P}_{-}, \mathcal{G}_{-}, \mathcal{Q}_{a}$, and $\lambda_{a}$ are odd (change the sign), while the rest of generators is even. Another set of transformations

$$
\begin{aligned}
& \rho_{2}: \quad \mathcal{H} \rightarrow \mathcal{K}, \quad \mathcal{K} \rightarrow \mathcal{H}, \quad \mathcal{D} \rightarrow-\mathcal{D}, \quad \mathcal{R} \rightarrow \mathcal{L}, \quad \mathcal{L} \rightarrow-\mathcal{R}, \\
& \mathcal{V} \rightarrow-\mathcal{G}, \quad \mathcal{G} \rightarrow \mathcal{V}, \quad \mathcal{G}_{-} \rightarrow \mathcal{P}_{-}, \quad \mathcal{P}_{-} \rightarrow-\mathcal{G}_{-}, \quad \Sigma \rightarrow \Sigma, \quad \mathcal{I} \rightarrow \mathcal{I}, \\
& \mathcal{Q}_{a} \rightarrow-\lambda_{a}, \quad \lambda_{a} \rightarrow \mathcal{Q}_{a}, \quad \mathcal{S}_{a} \rightarrow \kappa_{a}, \quad \kappa_{a} \rightarrow \mathcal{S}_{a}, \quad \mu_{a} \rightarrow-\mu_{a},
\end{aligned}
$$

corresponds to automorphism of the superalgebra that unifies and generalizes relations (3.6) and (3.21) for the superextended system (4.1).

\section{Symmetries of $\boldsymbol{H}_{n}^{\alpha}$ and of its super-extended version}

In this section we generalize the analysis of the previous two sections for the case of the $P T$-regularized conformal mechanics system $H_{n}^{\alpha}$ and its super-extended version. 
System (2.1) has the dynamical integrals of motion

$$
D_{n}^{\alpha}=-\frac{i}{2}\left((x+i \alpha) \frac{d}{d x}+\frac{1}{2}\right)-t H_{n}^{\alpha}, \quad K_{n}^{\alpha}=(x+i \alpha)^{2}-8 t D_{n}^{\alpha}-4 t^{2} H_{n}^{\alpha},
$$

which together with Hamiltonian $H_{n}^{\alpha}$ generate the conformal $s l(2, \mathbb{R})$ symmetry. It also has the Lax-Novikov integral of motion $P_{n}^{\alpha},\left[P_{n}^{\alpha}, H_{n}^{\alpha}\right]=0$, being the Darboux-dressed momentum integral $P_{0}$ of the free particle. Its scale dimension is $-\left(n+\frac{1}{2}\right), i\left[D_{n}^{\alpha}, P_{n}^{\alpha}\right]=-\left(n+\frac{1}{2}\right) P_{n}^{\alpha}$. Repeated commutation of $P_{n}^{\alpha}$ with $K_{n}^{\alpha},\left[P_{n}^{\alpha}, K_{n}^{\alpha}\right]$, $\left[\left[P_{n}^{\alpha}, K_{n}^{\alpha}\right], K_{n}^{\alpha}\right], \ldots$, generates in addition to $D_{n}^{\alpha}$ and $K_{n}^{\alpha}$, the $2 n$ dynamical integrals of the half-integer scale dimensions $-\left(n-\frac{1}{2}\right), \ldots,\left(n+\frac{1}{2}\right)$. These are the operators which together with the integral $P_{n}^{\alpha}$ can be presented in the form

$$
\mathcal{X}_{n, \ell}^{\alpha}=U_{n}^{-1}(t) X_{n, \ell}^{\alpha} U_{n}(t)=X_{n, \ell}^{\alpha}+\ldots, \quad \ell=0,1, \ldots, 2 n+1,
$$

where $U_{n}(t)=\exp \left(i t H_{n}^{\alpha}\right)$, the ellipsis corresponds to a polynomial of order $\ell$ in $t$,

$$
\begin{aligned}
& X_{n, \ell}^{\alpha}=(x+i \alpha)^{\ell} \mathcal{A}_{n, \ell}^{\alpha}, \\
& \mathcal{A}_{n, \ell}^{\alpha}=A_{\ell-n}^{\diamond} A_{\ell+1-n}^{\diamond} \ldots A_{n}^{\diamond}, \quad \ell=0,1, \ldots, 2 n, \quad \mathcal{A}_{n, 2 n+1}^{\alpha}=1,
\end{aligned}
$$

and we denote $A_{-k}^{\diamond}=A_{k}^{\alpha}$ for $k>0, A_{0}^{\diamond}=\frac{d}{d x}$, and $A_{k}^{\diamond}=A_{k}^{\alpha \#}$ for $k>0$. In particular cases of $\ell=0$ and $\ell=2 n+1$ the integrals (5.2) are reduced to $\mathcal{X}_{n, 0}=i P_{n}^{\alpha}$ and $\mathcal{X}_{n, 2 n+1}=$ $U_{n}^{-1}(t)(x+i \alpha)^{2 n+1} U_{n}(t)$. The integrals (5.2) form a $2(n+1)$-dimensional representation with respect to the adjoint action of the $\operatorname{sl}(2, \mathbb{R})$ generators,

$$
\begin{aligned}
& {\left[D_{n}^{\alpha}, \mathcal{X}_{n, \ell}^{\alpha}\right]=i\left(n-\ell+\frac{1}{2}\right) \mathcal{X}_{n, \ell}^{\alpha},} \\
& {\left[H_{n}^{\alpha}, \mathcal{X}_{n, \ell}^{\alpha}\right]=2 \ell \mathcal{X}_{n, \ell-1}^{\alpha}, \quad\left[K_{n}^{\alpha}, \mathcal{X}_{n, \ell}^{\alpha}\right]=2(2 n+1-\ell) \mathcal{X}_{n, \ell+1}^{\alpha} .}
\end{aligned}
$$

The commutation relations of $\mathcal{X}_{n, \ell}^{\alpha}$ between themselves are reduced to the form

$$
\left[\mathcal{X}_{n, \ell}^{\alpha}, \mathcal{X}_{n, \ell^{\prime}}^{\alpha}\right]=\mathcal{P}_{\ell, \ell^{\prime}}^{(2 n)}\left(H_{n}^{\alpha}, K_{n}^{\alpha}, D_{n}^{\alpha}\right)
$$

where $\mathcal{P}_{\ell, \ell^{\prime}}^{(2 n)}$ are some polynomials of order $2 n$ in the $\operatorname{sl}(2, \mathbb{R})$ generators.

The supersymmetric system given by the superpotential $\mathcal{W}_{e}=-n /(x+i \alpha)$ is described by the matrix Hamiltonian

$$
\mathcal{H}_{n}=\operatorname{diag}\left(H_{n}^{\alpha}, H_{n-1}^{\alpha}\right) .
$$

System (5.8) is characterized by nonlinear extension of the Schrödinger superalgebra, which in this case is generated by $4 n+6$ bosonic integrals and the same number of fermionic integrals, and by the bosonic central charge $\mathcal{I}$. The set of bosonic generators is given by $\mathcal{H}_{n}, \mathcal{D}_{n}=\operatorname{diag}\left(D_{n}^{\alpha}, D_{n-1}^{\alpha}\right), \mathcal{K}_{n}=\operatorname{diag}\left(K_{n}^{\alpha}, K_{n-1}^{\alpha}\right), \Sigma, \mathcal{X}_{n, \ell}^{+}=\mathcal{X}_{n, \ell}^{\alpha} \Pi_{+}, \ell=0, \ldots, 2 n+1$, and $\mathcal{X}_{n, k}^{-}=\mathcal{X}_{n-1, k}^{\alpha} \Pi_{-}, k=0, \ldots, 2 n-1$, where $\Pi_{ \pm}=\frac{1}{2}\left(1 \pm \sigma_{3}\right)$. The set of fermionic generators is $\mathcal{Q}_{n, a}, \lambda_{n, a}, \mathcal{S}_{n, a}$, and $\mu_{n, k, a}, k=0, \ldots, 2 n-1$. The explicit form of these 
generators with index $a=1$ is given by

$$
\begin{aligned}
\mathcal{Q}_{n, 1} & =\left(\begin{array}{cc}
0 & A_{n}^{-} \\
A_{n}^{\#} & 0
\end{array}\right), \quad \lambda_{n, 1}=\left(\begin{array}{cc}
0 & i(x+i \alpha) \\
-i(x+i \alpha) & 0
\end{array}\right)-2 t \mathcal{Q}_{n, 1}, \\
\mathcal{S}_{n, 1} & =\left(\begin{array}{cc}
0 & -i A_{n}^{\alpha} P_{n-1}^{\alpha} \\
i P_{n-1}^{\alpha} A_{n}^{\alpha \#} & 0
\end{array}\right), \\
\mu_{n, k, 1} & =\mathcal{U}_{n}^{-1}(t)\left(\begin{array}{cc}
0 & (x+i \alpha) X_{n-1, k}^{\alpha} \\
(-1)^{k+1} X_{n-1, k}^{\alpha}(x+i \alpha) & 0
\end{array}\right) \mathcal{U}_{n}(t) .
\end{aligned}
$$

The generators with index $a=2$ are obtained by multiplication with $i \sigma_{3}, \mathcal{Q}_{n, 2}=i \sigma_{3} \mathcal{Q}_{n, 1}$, etc., and here $\mathcal{U}_{n}(t)=\exp \left(i \mathcal{H}_{n} t\right)$ is the evolution operator. The operators $\mu_{n, k, a}$ with $k=$ $2 n-1$ and $k=n-1$ correspond, respectively, to the fermionic dynamical integrals (4.16) and (4.15) in the case of $n=1$.

The operators $\mathcal{H}_{n}, \mathcal{K}_{n}, \mathcal{D}_{n},\left(\mathcal{I}-\frac{1}{2} \Sigma\right), \mathcal{Q}_{n, a}$ and $\lambda_{n, a}$ are generators of the Lie subsuperalgebra $\operatorname{ssp}(2 \mid 2)$ of superconformal symmetry of the system (5.8). Extension of this set by any other integral (different from the central charge $\mathcal{I}$ ) results in expansion of the $o s p(2 \mid 2)$ up to the whole nonlinearly extended super-Schrödinger algebra. As in the $n=1$ case, the (anti)-commutators of all the integrals with the $\operatorname{ssp}(2 \mid 2)$ generators are linear in the generators of the extended super-Schrödinger algebra. Particularly, the operators $\mathcal{X}_{n, \ell}^{+}$and $\mathcal{X}_{n, k}^{-}$have the scaling dimensions $-\left(n-\ell+\frac{1}{2}\right)$ and $-\left(n-k-\frac{1}{2}\right)$, respectively, given by the adjoint action of $i \mathcal{D}_{n}$, while the pairs of fermionic operators $S_{n, a}$ and $\mu_{n, k, a}$, $a=1,2$, have the scaling dimensions $-n$ and $(k+1-n)$. In the (anti)-commutators of additional generators with generators of the $\operatorname{osp}(2 \mid 2)$ superconformal symmetry, the structure coefficients are certain polynomials of order $2 n-1$ in generators $\mathcal{H}, \mathcal{K}, \mathcal{D}$ and $\Sigma$ of the $\operatorname{sl}(2, \mathbb{R}) \oplus u(1)$ sub-algebra. The Hamiltonian $\mathcal{H}_{n}$, the supercharges $\mathcal{Q}_{n, a}$ and $\mathcal{S}_{n, a}$, and the bosonic Lax-Novikov matrix integral taken in the form $\mathcal{L}_{n}=\mathcal{X}_{n, 0}^{+}+\mathcal{H}_{n} \mathcal{X}_{n, 0}^{-}=$ $\operatorname{diag}\left(P_{n}^{\alpha}, H_{n-1}^{\alpha} P_{n-1}^{\alpha}\right)$ generate the exotic nonlinear $\mathcal{N}=4$ super-Poincaré algebra of the form (4.4), (4.5), (4.6), (4.7) with the unique difference in the anti-commutation relation between the higher order supercharges $\mathcal{S}_{n, a}:\left\{\mathcal{S}_{n, a}, \mathcal{S}_{n, b}\right\}=2 \delta_{a b}\left(\mathcal{H}_{n}\right)^{2 n}$. As in the particular case of $n=1$, the exotic nonlinear $\mathcal{N}=4$ supersymmetry of the system (5.8) is unbroken: its unique ground state of zero energy $\Psi^{t}=\left((x+i \alpha)^{-n}, 0\right)^{t}$ is annihilated by all the supercharges $\mathcal{Q}_{n, a}$ and $\mathcal{S}_{n, a}$ as well as by the Lax-Novikov integral [62]. Here, the operator $\mathcal{G}_{n}=\mathcal{X}_{n, 1}^{+}+\frac{1}{2}\left\{\mathcal{H}_{n}, \mathcal{X}_{n, 1}^{-}\right\}=\operatorname{diag}\left(\mathcal{X}_{n, 1}^{\alpha}, \frac{1}{2}\left\{\mathcal{H}_{n-1}, \mathcal{X}_{n-1,1}^{\alpha}\right\}\right)$ is the analog of the integral (4.10) of the case $n=1$.

\section{Spontaneoulsy broken phase of the exotic SUSY}

We study here the case of the $P T$-regularized $g=2$ superconformal mechanics model in the phase of the partially broken exotic nonlinear $\mathcal{N}=4$ super-Poincaré symmetry. The symmetry of the system we investigate is described by the same number of bosonic and fermionic generators as in the system from section 4 , but the structure of nonlinear superalgebra they generate is essentially different from that of the model (4.1). Nevertheless, its symmetry is shown may be related to the nonlinearly extended super-Schrödinger symmetry 
of the system (4.1) in the limit of the transition to the phase of the unbroken exotic nonlinear $\mathcal{N}=4$ supersymmetry.

The system we consider is generated via the $\mathcal{N}=2$ supersymmetric quantum mechanics construction based on the superpotential $\mathcal{W}_{b}(x)=1 / \xi_{1}-1 / \xi_{2}+i \delta^{-1}$, where $\xi_{j}=x+i \alpha_{j}$, $j=1,2, \mathbb{R} \ni \alpha_{j} \neq 0, \delta=\alpha_{1}-\alpha_{2} \neq 0$. In the limit when the parameter $\alpha_{1}$ (or $\alpha_{2}$ ) is sent to infinity, the superpotential transforms into the superpotential $\mathcal{W}_{e}(x)$ (or $\left.-\mathcal{W}_{e}(x)\right)$ of the system (4.1). We have $\mathcal{W}_{b}^{2}-\mathcal{W}_{b}^{\prime}=2 / \xi_{1}^{2}-\delta^{-2}, \mathcal{W}_{b}^{2}+\mathcal{W}_{b}^{\prime}=2 / \xi_{2}^{2}-\delta^{-2}$, that allows us to introduce the first order differential operators $A_{b}=\frac{d}{d x}+\mathcal{W}_{b}(x)$ and $A_{b}^{\#}=-\frac{d}{d x}+\mathcal{W}_{b}(x)$ satisfying the factorization, $A_{b}^{\#} A_{b}=H_{1}^{\alpha_{1}}-\delta^{-2}, A_{b} A_{b}^{\#}=H_{1}^{\alpha_{2}}-\delta^{-2}$, and the intertwining, $A_{b} H_{1}^{\alpha_{1}}=H_{1}^{\alpha_{2}} A_{b}, A_{b}^{\#} H_{1}^{\alpha_{2}}=H_{1}^{\alpha_{1}} A_{b}^{\#}$, relations, where $H_{1}^{\alpha_{j}}=-\frac{d^{2}}{d x^{2}}+2 / \xi_{j}^{2}$. Define now the extended system described by the matrix Hamiltonian

$$
\mathcal{H}=\operatorname{diag}\left(H_{1}^{\alpha_{2}}, H_{1}^{\alpha_{1}}\right) .
$$

Operators

$$
\mathcal{Q}_{1}=\left(\begin{array}{cc}
0 & A_{b} \\
A_{b}^{\#} & 0
\end{array}\right), \quad \mathcal{Q}_{2}=i \sigma_{3} \mathcal{Q}_{1},
$$

are the supercharges of the system (6.1). They satisfy the relations

$$
\left[\mathcal{H}, \mathcal{Q}_{a}\right]=0, \quad\left\{\mathcal{Q}_{a}, \mathcal{Q}_{b}\right\}=2 \delta_{a b}\left(\mathcal{H}-\delta^{-2}\right) .
$$

The partner Hamiltonians can also be intertwined by the second order operators $A_{1}^{\alpha_{2}} A_{1}^{\alpha_{1} \#}$ and $A_{1}^{\alpha_{1}} A_{1}^{\alpha_{2} \#}$ via the Hamiltonian of the intermediate ('virtual' here) free particle system : $A_{1}^{\alpha_{2}} A_{1}^{\alpha_{1} \#} H_{1}^{\alpha_{1}}=A_{1}^{\alpha_{2}} H_{0} A_{1}^{\alpha_{1} \#}=H_{1}^{\alpha_{2}} A_{1}^{\alpha_{2}} A_{1}^{\alpha_{1} \#}, A_{1}^{\alpha_{1}} A_{1}^{\alpha_{2} \#} H_{1}^{\alpha_{2}}=A_{1}^{\alpha_{1}} H_{0} A_{1}^{\alpha_{2} \#}=$ $H_{1}^{\alpha_{1}} A_{1}^{\alpha_{1}} A_{1}^{\alpha_{2} \#}$. Hence, system (6.1) is characterized additionally by the second order supercharges

$$
\begin{aligned}
& \mathcal{S}_{1}=\left(\begin{array}{cc}
0 & A_{1}^{\alpha_{2}} A_{1}^{\alpha_{1} \#} \\
A_{1}^{\alpha_{1}} A_{1}^{\alpha_{2} \#} & 0
\end{array}\right), \quad \mathcal{S}_{2}=i \sigma_{3} \mathcal{S}_{1}, \\
& {\left[\mathcal{H}, \mathcal{S}_{a}\right]=0, \quad\left\{\mathcal{S}_{a}, \mathcal{S}_{b}\right\}=2 \delta_{a b} \mathcal{H}^{2} .}
\end{aligned}
$$

We note here that the first order intertwining operators from which supercharges (6.2) are composed can be obtained by employing the reduction of intertwining operators mentioned in section 4. Indeed, the Hamiltonians $H_{1}^{\alpha_{1}}$ and $H_{1}^{\alpha_{2}}$ can be intertwined by the third order differential operator $A_{1}^{\alpha_{2}} P_{0} A_{1}^{\alpha_{1} \#}$ by using the chain of equalities $A_{1}^{\alpha_{2}} P_{0} A_{1}^{\alpha_{1} \#} H_{1}^{\alpha_{1}}=$ $A^{\alpha_{2}} P_{0} H_{0} A_{1}^{\alpha_{1} \#}=A_{1}^{\alpha_{2}} H_{0} P_{0} A_{1}^{\alpha_{1} \#}=H_{1}^{\alpha_{2}} A_{1}^{\alpha_{2}} P_{0} A_{1}^{\alpha_{1} \#}$. However, using equation (4.18) from ref. [62], or by a direct computation, one finds the relation $A_{1}^{\alpha_{2}} P_{0} A_{1}^{\alpha_{1} \#}=H_{1}^{\alpha_{2}} A_{b}-$ $i \delta^{-1} A_{1}^{\alpha_{2}} A_{1}^{\alpha_{1} \#}$ that shows that the indicated third order intertwining operator reduces to the intertwining operators appearing in the right-upper corners of the supercharges $\mathcal{Q}_{1}$ and $\mathcal{S}_{1}$ multiplied, respectively, by the second order operator $H_{1}^{\alpha_{2}}$ and the constant $-i \delta^{-1}$.

The anti-commutator of the first and second order supercharges generates the matrix Lax-Novikov integral $\mathcal{L}_{1}=\operatorname{diag}\left(P_{1}^{\alpha_{2}}, P_{1}^{\alpha_{1}}\right)$,

$$
\left\{\mathcal{Q}_{a}, \mathcal{S}_{b}\right\}=2\left(\epsilon_{a b} \mathcal{L}_{1}+i \delta_{a b} \delta^{-1} \mathcal{H}\right)
$$


This operator satisfies relations $\left[\mathcal{L}_{1}, \mathcal{H}\right]=\left[\mathcal{L}_{1}, \mathcal{Q}_{a}\right]=\left[\mathcal{L}_{1}, \mathcal{S}_{a}\right]=0$, and plays the role of the central charge of the exotic nonlinear $\mathcal{N}=4$ supersymmetry generated by $\mathcal{H}$, $\mathcal{Q}_{a}, \mathcal{S}_{a}$ and $\mathcal{L}_{1}$. Unlike (4.1), system (6.1) is in the phase of the partially broken exotic nonlinear $\mathcal{N}=4$ supersymmetry. Its two zero energy eigenstates $\Psi_{\alpha_{2}}^{t}=\left(\left(x+i \alpha_{2}\right)^{-1}, 0\right)^{t}$ and $\Psi_{\alpha_{1}}^{t}=\left(0,\left(x+i \alpha_{1}\right)^{-1}\right)^{t}$ are zero modes of both second order supercharges $\mathcal{S}_{a}$, but neither of them is annihilated by the first order supercharges $\mathcal{Q}_{a}$ [62].

System (6.1) is also characterized by the dynamical integrals $\mathcal{D}=\operatorname{diag}\left(D_{1}^{\alpha_{2}}, D_{1}^{\alpha_{1}}\right)$ and $\mathcal{K}=\operatorname{diag}\left(K_{1}^{\alpha_{2}}, K_{1}^{\alpha_{1}}\right)$. Commuting the Lax-Novikov integral repeatedly with the dynamical integral $\mathcal{K}$, we generate three more bosonic dynamical integrals of motion,

$$
\begin{array}{rlrl}
{\left[\mathcal{L}_{1}, \mathcal{K}\right]} & =-6 i \mathcal{G}_{1}, \quad\left[\mathcal{G}_{1}, \mathcal{K}\right] & =-4 i \mathcal{V}, \quad[\mathcal{V}, \mathcal{K}]=-2 i \mathcal{R}, \quad[\mathcal{R}, \mathcal{K}]=0 \\
\mathcal{G}_{1} & =\left(\Xi \mathcal{H}+\mathcal{A}^{\#}\right)-2 t \mathcal{L}_{1}, \quad \mathcal{V}=i \Xi^{2} \mathcal{A}^{\#}-4 t \mathcal{G}_{1}-4 t^{2} \mathcal{L}_{1}, \\
\mathcal{R} & =\Xi^{3}-6 t \mathcal{V}-12 t^{2} \mathcal{G}_{1}-8 t^{3} \mathcal{L}_{1}
\end{array}
$$

where $\Xi=\operatorname{diag}\left(\xi_{2}, \xi_{1}\right), \mathcal{A}^{\#}=\operatorname{diag}\left(A_{1}^{\alpha_{2} \#}, A_{1}^{\alpha_{1} \#}\right)$. All these bosonic integrals have the same scaling dimensions as their analogs in the system from section 3 . The peculiarity of the system (6.1), however, is that its supercharges $\mathcal{Q}_{a}$ and $\mathcal{S}_{a}$ are not eigenstates of the dilatation generator under its adjoint action:

$$
\left[\mathcal{D}, \mathcal{Q}_{a}\right]=\frac{1}{2} \delta \mathcal{S}_{a}, \quad\left[\mathcal{D}, \mathcal{S}_{a}\right]=\frac{3}{2} i \mathcal{S}_{a}-\frac{1}{2} \delta \mathcal{H} \mathcal{Q}_{a}
$$

Coherently with this, the way in which the odd dynamical integrals for the system (6.1) are generated is also different in comparison with that for the system from section 4 . The commutation of the dynamical integral $\mathcal{K}$ with the first and second order supercharges $\mathcal{Q}_{a}$ and $\mathcal{S}_{a}$ generates the unique pair of the odd dynamical integrals $\mu_{a}$ :

$$
\begin{aligned}
{\left[\mathcal{K}, \mathcal{Q}_{a}\right] } & =\delta^{2} \mathcal{Q}_{a}+2 \delta \mu_{a}, & {\left[\mathcal{K}, \mathcal{S}_{a}\right] } & =\delta^{2} \mathcal{S}_{a}+3 i \delta \mathcal{Q}_{a}-4 \delta \mathcal{D} \mathcal{Q}_{a}+6 i \mu_{a} \\
\mu_{1} & =i \Xi \sigma_{1} \mathcal{A}^{\#}-2 t \mathcal{S}_{1}, & \mu_{2} & =i \sigma_{3} \mu_{1} .
\end{aligned}
$$

Commutation of $\mathcal{K}$ with $\mu_{a}$ produces a new pair of fermionic integrals $\Gamma_{a}$,

$$
\begin{aligned}
{\left[\mathcal{K}, \mu_{a}\right] } & =4 i \Gamma_{a}+\delta^{2} \mu_{a}-2 \delta \mathcal{K} \mathcal{Q}_{a}, \\
\Gamma_{1} & =\left(\Xi^{2} \sigma_{1}-\delta \Xi \sigma_{2}\right)-\left(4 \mu_{1}+2 \delta Q_{1}\right) t-4 t^{2} S_{1}, \quad \Gamma_{2}=i \sigma_{3} \Gamma_{1} .
\end{aligned}
$$

The time-independent term in (6.14) is presented equivalently in the form $\xi_{1} \xi_{2} \sigma_{1}$. Finally, commutation of $\mathcal{K}$ with $\Gamma_{a}$ generates dynamical odd integrals $\Omega_{a}$,

$$
\begin{aligned}
{\left[\mathcal{K}, \Gamma_{a}\right]=} & \Omega_{a} \\
\Omega_{1}= & \left(3 \delta^{2} \Xi^{2} \sigma_{1}+\left(2 \delta \Xi^{3}-\delta^{3} \Xi\right) \sigma_{2}\right)+t\left(-2 \delta^{3} \mathcal{Q}_{1}-12 \delta^{2} \mu_{1}+12 \delta \mathcal{K} \mathcal{Q}_{1}-12 i \Gamma_{1}\right) \\
& +t^{2}\left(-12 \delta^{2} \mathcal{S}_{1}+48 \delta \mathcal{D} Q_{1}-24 i \mu_{1}\right)+t^{3}\left(-16 i \mathcal{S}_{1}+16 \delta \mathcal{H} \mathcal{Q}_{1}\right)
\end{aligned}
$$

$\Omega_{2}=i \sigma_{3} \Omega_{1}$. The commutation relation

$$
\left[\mathcal{K}, \Omega_{a}\right]=\delta^{2}\left(4 \Omega_{a}-2 \delta^{2} \Gamma_{a}-\frac{1}{2} \mathcal{K} \Gamma_{a}\right)
$$


then signals that the process of generation of odd dynamical integrals terminates. Commutation relations

$$
\begin{aligned}
& {\left[\mathcal{D}, \mu_{a}\right]=\frac{i}{2} \mu_{a}-\delta\left(\mathcal{D}-\frac{i}{4}\right) \mathcal{Q}_{a}, \quad\left[\mathcal{D}, \Gamma_{a}\right]=-\frac{i}{2} \Gamma_{a}-\frac{1}{2} \delta \mathcal{K} \mathcal{Q}_{a}+\frac{1}{2} \delta^{2} \mu_{a}} \\
& {\left[\mathcal{D}, \Omega_{a}\right]=-\frac{3}{2} i \Omega_{a}-\frac{3}{2} \delta^{3} \mathcal{K} \mathcal{Q}_{a}+2 i \delta^{2} \Gamma_{a}+\delta^{2}\left(\frac{1}{2} \delta^{2}-\mathcal{K}\right) \mu_{a}}
\end{aligned}
$$

show that, like $\mathcal{Q}_{a}$ and $\mathcal{S}_{a}$, neither of the odd dynamical integrals is eigenstate under the adjoint action of the dilation generator. The commutation relations of the odd dynamical integrals with the Hamiltonian operator are

$$
\begin{aligned}
{\left[\mathcal{H}, \mu_{a}\right] } & =-2 i S_{a}, \quad\left[\mathcal{H}, \Gamma_{a}\right]=-2 i\left(2 \mu_{a}+\delta Q_{a}\right) \\
{\left[\mathcal{H}, \Omega_{a}\right] } & =12 \Gamma_{a}-i \delta^{2}\left(10 \mu_{a}+3 \delta Q_{a}\right)+4 \delta^{2} \mathcal{D}\left(\mu_{a}+\delta Q_{a}\right)+12 i \mathcal{K} Q_{a}
\end{aligned}
$$

The anti-commutation relations of dynamical integrals $\mu_{a}$ with $\mu_{b}, \mathcal{Q}_{b}$ and $\mathcal{S}_{b}$ are

$$
\begin{aligned}
\left\{\mu_{a}, \mu_{b}\right\} & =8 \delta_{a b}\left(\mathcal{D}^{2}-4 i \mathcal{D}+2 i \delta \mathcal{G}_{2}-9 \mathcal{I}\right) \\
\left\{\mathcal{Q}_{a}, \mu_{b}\right\} & =\delta_{a b}\left(-\delta \mathcal{H}+4 i \delta^{-1} \mathcal{D}+\delta^{-1} \mathcal{I}\right)+\epsilon_{a b}\left(2 \mathcal{G}_{1}+i \delta \Sigma \mathcal{H}\right) \\
\left\{\mathcal{S}_{a}, \mu_{b}\right\} & =\delta_{a b}\left(-3 i \mathcal{H}+4 \mathcal{D} \mathcal{H}+i \delta \mathcal{L}_{2}\right)+\epsilon_{a b}\left(-2 \Sigma \mathcal{H}+\delta \mathcal{L}_{1}\right)
\end{aligned}
$$

where $\mathcal{L}_{2}=\sigma_{3} \mathcal{L}_{1}$ and $\mathcal{G}_{2}=\sigma_{3} \mathcal{G}_{1}$ are two additional bosonic integrals. Instead of them, one can take their linear combinations with $\mathcal{L}_{1}$ and $\mathcal{G}_{1}$ to obtain $\mathcal{L}_{-}=\Pi_{-} \mathcal{L}_{1}$ and $\mathcal{G}_{-}=\Pi_{-} \mathcal{G}_{1}$, which could be considered as analogs of the integrals (4.13) of the system (4.1). Finally, the system under consideration is described by ten bosonic integrals $\mathcal{H}, \mathcal{D}, \mathcal{K}, \Sigma, \mathcal{L}_{1}, \mathcal{G}_{1}$, $\mathcal{V}, \mathcal{R}, \mathcal{L}_{2}$ and $\mathcal{G}_{2}$ (or by $\mathcal{L}_{-}$and $\mathcal{G}_{-}$instead of the two last integrals), the same number of fermionic integrals $\mathcal{Q}_{a}, \mathcal{S}_{a}, \mu_{a}, \Gamma_{a}$ and $\Omega_{a}$, and by the central charge $\mathcal{I}$.

Essential peculiarity here is that the $P T$-regularization parameter $\alpha$ of the dimension of length does not appear in the superalgebra of the system (4.1), while in the (anti)commutation relations for the system (6.1) there appears the parameter $\delta=\alpha_{1}-\alpha_{2}$.

We will not write down the missing (anti)-commutation relations of the integrals, but examine the relation between the systems (4.1) and (6.1) in the light of their nonlinearly supersymmetrically extended and deformed conformal symmetries. First of all, we observe that the nonlinear extensions of the conformal $s l(2, \mathbb{R})$ symmetry generated by their sets of the bosonic integrals are very similar. The only difference appears in commutation relations of the corresponding pairs of the operators $\left(\mathcal{P}_{-}, \mathcal{G}_{-}\right)$and $\left(\mathcal{L}_{-}, \mathcal{G}_{-}\right)$. More essential difference is that system (4.1) contains the algebra of the superconformal $o s p(2 \mid 2)$ symmetry as a sub-superalgebra, whereas system (6.1) does not have such a subsuperalgebra. This is reflected, particularly, in the peculiarity of the latter system encoded in relations (6.10), (6.18) and (6.19), cf. (4.21). Since in the limit $\alpha_{1} \rightarrow \infty$ with identification $\alpha_{2}=\alpha$ the superpotential $\mathcal{W}_{b}(x)$ of the system (6.1) transforms into superpotential $\mathcal{W}_{e}(x)$ of the system (4.1), and the Hamiltonian (6.1) transforms into (4.1), the essential difference between two systems at first glance may seem to be rather surprising. To clarify this point, let us see what happens in the indicated limit with other operators. We have 
$A_{b} \rightarrow A_{1}^{\alpha}, A_{b}^{\#} \rightarrow A_{1}^{\alpha \#}, A_{1}^{\alpha_{1}} \rightarrow \frac{d}{d x}$ and $A_{1}^{\alpha_{1} \#} \rightarrow-\frac{d}{d x}$. As a result, we find that the generators $\mathcal{Q}_{a}, \mathcal{S}_{a}$ and $\mathcal{L}_{1}$ of the system (6.1) transform into the corresponding integrals $\mathcal{Q}_{a}$, $\mathcal{S}_{a}$ and $\mathcal{L}$ of the system (4.1), while $\mathcal{L}_{-}$transforms into the integral $\mathcal{P}_{-}$multiplied with the Hamiltonian (4.1). The limit applied to other integrals is more delicate, however. The dilatation generator of the system (6.1) with $\alpha_{2}=\alpha$ is represented equivalently in the form $\mathcal{D}=\mathcal{D}(x+i \alpha)+\frac{i}{2}\left(\alpha_{1}-\alpha\right) \mathcal{P}_{-}$, where by $\mathcal{D}(x+i \alpha)$ we indicate the dilatation generator for the system (4.1). So, the integral $\mathcal{D}$ transforms into $\mathcal{D}(x+i \alpha)$ if the limit $\alpha_{1} \rightarrow \infty$ is accompanied by a 'renormalization' which consists in omitting the integral $\frac{i}{2} \mathcal{P}$ - multiplied with the factor $\delta=\left(\alpha_{1}-\alpha\right) \rightarrow \infty$. A similar picture is valid for relation between other bosonic dynamical integrals of both systems in the indicated limit. Particularly, we have $\mathcal{K}=\mathcal{K}(x+i \alpha)+2 i \delta \mathcal{J}_{-}(x+i \alpha)-\delta^{2} \Pi_{-}$, and this integral transforms into $\mathcal{K}(x+i \alpha)$ by making the same kind of 'renormalization'. For fermionic dynamical integral $\mu_{1}$ the relation $\mu_{1}=\mu_{1}(x+\alpha)-\delta \Pi_{-} \mathcal{Q}_{1}$ is valid when $\alpha_{1} \rightarrow \infty$, and, as a result, a 'renormalized' form of the integrals (6.12) will correspond to the dynamical integrals (4.15) of the system (4.1). For the integral $\Gamma_{1}$ with $\alpha_{1} \rightarrow \infty$ we have $\Gamma_{1}=\kappa_{1}(x+i \alpha)-i \delta \lambda_{2}(x+i \alpha)$, and the 'renormalized' form of the integrals (6.14) gives us the integrals (4.16) for the system (4.1). This also means that the limit $\alpha_{1} \rightarrow \infty$ applied to the rescaled integrals $\delta^{-1} \Gamma_{a}$ reproduces the dynamical integrals (5.10). Analogously, the appropriately rescaled integrals (6.16) in the limit $\alpha_{1} \rightarrow \infty$ reproduce the integrals (5.10): $\delta^{-3} \Omega_{a} \rightarrow \lambda_{a}(x+i \alpha)$.

All this shows that in the limit when one of the two parameters $\alpha_{1}$ or $\alpha_{2}$ is sent to infinity, the system (6.1) in the phase of the partially broken exotic nonlinear $\mathcal{N}=4$ superPoincaré symmetry transforms into the system (4.1) in the unbroken phase, and all the integrals of the latter system can be reproduced from those of the former system. The relation between the integrals, however, is rather non-trivial, that is behind the essential difference between the nonlinear super-extended versions of the conformal symmetry generated in two cases.

\section{Discussion and outlook}

The AFF model [1] is obtained from the model (1.1) by adding into the Hamiltonian operator the confining harmonic potential term $x^{2}$ that effectively results in introduction of another boundary condition $\psi(+\infty)=0$ at another edge of the interval $x \in(0,+\infty)$ in addition to the Dirichlet boundary condition $\psi(0)=0$. This procedure does not change the symmetry: the system described by the "regularized" AFF Hamiltonian has the same conformal symmetry as the initial system (1.1), but it changes radically the spectrum. Instead of continuous non-degenerate spectrum of the system (1.1) with $E>0$, the AFF model has the equidistant discrete spectrum which, up to a constant shift, coincides with the spectrum of the half-harmonic oscillator [94]. This is not surprising as at particular values of the coupling constant $g=n(n+1)$, the AFF model is generated from the half-harmonic oscillator by the appropriate nonsingular on the half-line Darboux-Crum transformation [94]. From this point of view the AFF model with confining harmonic potential term is rather a rational deformation of the half-harmonic oscillator than the deformation (by adding the harmonic term) of the two-particle Calogero system (1.1) with the omitted center of mass 
coordinate. Nevertheless, in this way the "regularization" recipe accepted in [1] solves the problem of the absence of the ground state in the initial system (1.1). One could not restrict the values of $x$ to the half-line in the system (1.1) as well as in the "regularized" AFF model and consider both systems on the whole real line $x \in \mathbb{R}$. Potential with the inverse squared term is not penetrable at $x=0$, and the probability flux between regions $x<0$ and $x>0$ will be equal to zero, i.e. in this case we effectively will have two copies of the system with doubly degenerate either continuous spectrum with $E>0$ in the case of (1.1) or the discrete spectrum in the AFF model [1]. Besides the conformal symmetry, system (1.1) has another very important peculiarity. As we noted, it's potential with $g=n(n+1)$ is an algebro-geometric solution to the stationary KdV equation or the higher equation of its hierarchy, which is characterized by the existence of the differential operator of order $2 n+1$, related with a higher order Novikov equation [96]. This higher order Lax-Novikov operator commutes with the Hamiltonian operator (1.1) [71]. In this picture, a free particle on the whole real line can be treated as a zero-gap $(n=0)$ system for which the corresponding first order Lax-Novikov differential operator is just the momentum integral $-i \frac{d}{d x}$. But unlike the free particle case with $x \in \mathbb{R}$, the Lax-Novikov differential operator of order $2 n+1$ for the quantum system (1.1) with $g=n(n+1)$ is not a true integral of motion since it takes out the wave functions from the domain of the Hamiltonian operator (1.1), as it also happens for the operator $-i \frac{d}{d x}$ for the free particle on the half-line. The PT-regularization of the system (1.1) we apply, $x \rightarrow x+i \alpha, \mathbb{R} \ni \alpha \neq 0$, $x>0 \rightarrow x \in \mathbb{R}$, allows us to transform the Lax-Novikov operator of the algebro-geometric method of solution of the KdV equation and higher equations of its hierarchy, into the true integral for the quantum system $H_{n}^{\alpha}=-\frac{d^{2}}{d x^{2}}+\frac{n(n+1)}{(x+i \alpha)^{2}}$, which turns out to be a Darbouxdressed momentum of the free particle on the whole line. As a result, the symmetry of the PT-regularized system is described by nonlinearly extended generalized Shrödinger algebra which includes conformal $s l(2, \mathbb{R})$ algebra as the subalgebra, and there appears a non-degenerate bound state $\psi_{0}=1 /(x+i \alpha)^{n}$ of zero energy at the very edge of its doubly degenerate continuous spectrum, which is nothing else as the Darboux-Crum transformed zero energy free particle's state $\psi_{0}=1$. As we showed, such extension of symmetry also has very profound consequences for supersymmetric $P T$-regularized conformal mechanics systems in comparison with the superconformal $\operatorname{ssp}(2 \mid 2)$ symmetry of the superextended version of the system (1.1). Particularly, in the $P T$-regularized superconformal mechanics system in the partially broken phase of the exotic nonlinear $\mathcal{N}=4$ super-Poincaré symmetry we considered in the previous section, the $\operatorname{osp}(2 \mid 2)$ is not contained at all as a sub-superalgebra in the corresponding nonlinearly super-extended Schrödinger algebra.

As it was shown in [62], the systems we considered here are the simplest representatives of a broader class of the systems which can be generated by applying the chains of Darboux transformations to the quantum free particle. The interesting question is what happens with the conformal symmmetry for more complicated systems from the indicated class, particularly, in the systems related to solutions of the equations of the KdV hierarchy which reveal the properties of the extreme waves [62].

Since conformal mechanics (1.1) with special values of the coupling constant $g=$ $n(n+1)$ plays a special role in the Huygens' principle [68], the interesting question that 
deserves a separate investigation corresponds to the treatment of the considered systems as (1+1)-dimensional field theories from the perspective of the associated time-dependent Schrödinger equation. An a priori complication which may appear in this direction is that due to the higher-derivative nature of the Lax-Novikov operators, it is impossible, at least directly, to associate them with Noether integrals of the corresponding field systems. The indicated field-theoretical generalization is also interesting bearing in mind that the considered $P T$-symmetric quantum mechanical systems are also related to the singular kinks arising as traveling waves in the Liouville and SU(3) conformal Toda systems [62].

The obtained results could be generalized for the case of the AFF conformal mechanics model with the confining harmonic potential term. The essential difference in such a case is that the corresponding systems do not have Lax-Novikov integrals. However, in the case of rational deformations of such systems with special values of the coupling constant $g=n(n+1)$, instead of the Lax-Novikov integrals they are characterized by higherderivative spectrum-generating ladder operators [93, 94]. As a result, in that case also there appear nonlinear extensions of the (super)-Schrödinger symmetry of the structure similar to that investigated here [97].

It would be interesting to generalize our results for the case of appropriately PTregularized many-particle superconformal mechanics [7]-[14]. There, additional integrals also can be constructed in the form of higher-order Lax-Novikov type integrals via the Darboux-dressing procedure [98]. It is necessary to stress that the formal Lax-Novikov type integrals in $n$-particle Calogero-Moser systems with $n>2$ also have a pure quantum nature, and they are not related to (maximal) super-integrability of the systems which takes place already at the classical level [99-102]. Similarly to the present case of $n=2$, the square of the indicated formal integrals also reduces there to a polynomial in the Liouville charges [98].

\section{Acknowledgments}

We acknowledge support from research projects FONDECYT 1130017 and Convenio Marco Universidades del Estado (Project USA1555), Chile, and MINECO (Project MTM201457129- C2-1-P), Spain. JMG also acknowledges the Junta de Castilla y León for financial support under grant VA057U16. MSP is grateful for the warm hospitality at Salamanca University where this work started.

Open Access. This article is distributed under the terms of the Creative Commons Attribution License (CC-BY 4.0), which permits any use, distribution and reproduction in any medium, provided the original author(s) and source are credited.

\section{References}

[1] V. de Alfaro, S. Fubini and G. Furlan, Conformal Invariance in Quantum Mechanics, Nuovo Cim. A 34 (1976) 569 [InSPIRE].

[2] F. Calogero, Solution of the one-dimensional $N$ body problems with quadratic and/or inversely quadratic pair potentials, J. Math. Phys. 12 (1971) 419 [INSPIRE]. 
[3] V.P. Akulov and A.I. Pashnev, Quantum superconformal model in $(1,2)$ space, Theor. Math. Phys. 56 (1983) 862 [InSPIRE].

[4] S. Fubini and E. Rabinovici, Superconformal quantum mechanics, Nucl. Phys. B 245 (1984) 17 [INSPIRE].

[5] E.A. Ivanov, S.O. Krivonos and V.M. Leviant, Geometry of Conformal Mechanics, J. Phys. A 22 (1989) 345 [INSPIRE].

[6] E.A. Ivanov, S.O. Krivonos and V.M. Leviant, Geometric superfield approach to superconformal mechanics, J. Phys. A 22 (1989) 4201 [INSPIRE].

[7] D.Z. Freedman and P.F. Mende, An Exactly Solvable N Particle System in Supersymmetric Quantum Mechanics, Nucl. Phys. B 344 (1990) 317 [InSPIRE].

[8] N. Wyllard, (Super)conformal many body quantum mechanics with extended supersymmetry, J. Math. Phys. 41 (2000) 2826 [hep-th/9910160] [INSPIRE].

[9] S. Bellucci, A. Galajinsky, E. Ivanov and S. Krivonos, $A d S_{2} / C F T_{1}$, canonical transformations and superconformal mechanics, Phys. Lett. B 555 (2003) 99 [hep-th/0212204] [INSPIRE].

[10] S. Bellucci, A. Galajinsky and S. Krivonos, New many-body superconformal models as reductions of simple composite systems, Phys. Rev. D 68 (2003) 064010 [hep-th/0304087] [INSPIRE].

[11] S. Bellucci, A.V. Galajinsky and E. Latini, New insight into WDVV equation, Phys. Rev. D 71 (2005) 044023 [hep-th/0411232] [INSPIRE].

[12] A. Galajinsky, O. Lechtenfeld and K. Polovnikov, Calogero models and nonlocal conformal transformations, Phys. Lett. B 643 (2006) 221 [hep-th/0607215] [INSPIRE].

[13] A. Galajinsky, O. Lechtenfeld and K. Polovnikov, $N=4$ superconformal Calogero models, JHEP 11 (2007) 008 [arXiv:0708.1075] [INSPIRE].

[14] S. Krivonos and O. Lechtenfeld, Many-particle mechanics with $D(2,1: \alpha)$ superconformal symmetry, JHEP 02 (2011) 042 [arXiv:1012.4639] [INSPIRE].

[15] J.M. Maldacena, The Large $N$ limit of superconformal field theories and supergravity, Int. J. Theor. Phys. 38 (1999) 1113 [hep-th/9711200] [INSPIRE].

[16] S.S. Gubser, I.R. Klebanov and A.M. Polyakov, Gauge theory correlators from noncritical string theory, Phys. Lett. B 428 (1998) 105 [hep-th/9802109] [INSPIRE].

[17] E. Witten, Anti-de Sitter space and holography, Adv. Theor. Math. Phys. 2 (1998) 253 [hep-th/9802150] [INSPIRE].

[18] P. Claus, M. Derix, R. Kallosh, J. Kumar, P.K. Townsend and A. Van Proeyen, Black holes and superconformal mechanics, Phys. Rev. Lett. 81 (1998) 4553 [hep-th/9804177] [INSPIRE].

[19] J.A. de Azcarraga, J.M. Izquierdo, J.C. Perez Bueno and P.K. Townsend, Superconformal mechanics and nonlinear realizations, Phys. Rev. D 59 (1999) 084015 [hep-th/9810230] [INSPIRE].

[20] G.W. Gibbons and P.K. Townsend, Black holes and Calogero models, Phys. Lett. B 454 (1999) 187 [hep-th/9812034] [INSPIRE].

[21] J. Michelson and A. Strominger, Superconformal multiblack hole quantum mechanics, JHEP 09 (1999) 005 [hep-th/9908044] [INSPIRE]. 
[22] G.F. de Teramond, H.G. Dosch and S.J. Brodsky, Baryon Spectrum from Superconformal Quantum Mechanics and its Light-Front Holographic Embedding, Phys. Rev. D 91 (2015) 045040 [arXiv: 1411.5243] [INSPIRE].

[23] S.J. Brodsky, G.F. de Téramond, H.G. Dosch and C. Lorcé, Universal Effective Hadron Dynamics from Superconformal Algebra, Phys. Lett. B 759 (2016) 171 [arXiv:1604.06746] [INSPIRE].

[24] E. D'Hoker and L. Vinet, Dynamical Supersymmetry of the Magnetic Monopole and the 1/r $r^{2}$ Potential, Commun. Math. Phys. 97 (1985) 391 [InSPIRE].

[25] J. Michelson and A. Strominger, The Geometry of (super)conformal quantum mechanics, Commun. Math. Phys. 213 (2000) 1 [hep-th/9907191] [INSPIRE].

[26] S. Cacciatori, D. Klemm and D. Zanon, $W_{\infty}$ algebras, conformal mechanics and black holes, Class. Quant. Grav. 17 (2000) 1731 [hep-th/9910065] [INSPIRE].

[27] R. Britto-Pacumio, J. Michelson, A. Strominger and A. Volovich, Lectures on Superconformal Quantum Mechanics and Multi-Black Hole Moduli Spaces, NATO Sci. Ser. C 556 (2000) 255 [hep-th/9911066] [InSPIRE].

[28] G. Papadopoulos, Conformal and superconformal mechanics, Class. Quant. Grav. 17 (2000) 3715 [hep-th/0002007] [INSPIRE].

[29] E.E. Donets, A. Pashnev, V.O. Rivelles, D.P. Sorokin and M. Tsulaia, $N=4$ superconformal mechanics and the potential structure of AdS spaces, Phys. Lett. B 484 (2000) 337 [hep-th/0004019] [INSPIRE].

[30] M.S. Plyushchay, Monopole Chern-Simons term: Charge monopole system as a particle with spin, Nucl. Phys. B 589 (2000) 413 [hep-th/0004032] [INSPIRE].

[31] P.K. Ghosh, Conformal symmetry and the nonlinear Schrödinger equation, Phys. Rev. A 65 (2002) 012103 [cond-mat/0102488] [INSPIRE].

[32] M. Günaydin, K. Koepsell and H. Nicolai, The Minimal unitary representation of $E_{8(8)}$, Adv. Theor. Math. Phys. 5 (2002) 923 [hep-th/0109005] [InSPIRE].

[33] B. Pioline and A. Waldron, Quantum cosmology and conformal invariance, Phys. Rev. Lett. 90 (2003) 031302 [hep-th/0209044] [INSPIRE].

[34] H.E. Camblong and C.R. Ordonez, Anomaly in conformal quantum mechanics: From molecular physics to black holes, Phys. Rev. D 68 (2003) 125013 [hep-th/0303166] [INSPIRE].

[35] C. Leiva and M.S. Plyushchay, Conformal symmetry of relativistic and nonrelativistic systems and AdS/CFT correspondence, Annals Phys. 307 (2003) 372 [hep-th/0301244] [INSPIRE].

[36] B. Pioline and A. Waldron, Automorphic forms: A Physicist's survey, in Proceedings, Les Houches School of Physics: Frontiers in Number Theory, Physics and Geometry II: On Conformal Field Theories, Discrete Groups and Renormalization, Les Houches, France, March 9-21, 2003, pp. 277-302, (2007) [DOI:10.1007/978-3-540-30308-4_7] [hep-th/0312068] [INSPIRE].

[37] D. Gaiotto, A. Strominger and X. Yin, Superconformal black hole quantum mechanics, JHEP 11 (2005) 017 [hep-th/0412322] [INSPIRE].

[38] S. Meljanac and A. Samsarov, Universal properties of conformal quantum many-body systems, Phys. Lett. B 613 (2005) 221 [Erratum ibid. B 620 (2005) 200] [hep-th/0503174] [INSPIRE]. 
[39] C. Duval, G.W. Gibbons and P. Horvathy, Celestial mechanics, conformal structures and gravitational waves, Phys. Rev. D 43 (1991) 3907 [hep-th/0512188] [INSPIRE].

[40] A. Anabalon, J. Gomis, K. Kamimura and J. Zanelli, $N=4$ superconformal mechanics as a non linear realization, JHEP 10 (2006) 068 [hep-th/0607124] [INSPIRE].

[41] M. Günaydin, A. Neitzke, B. Pioline and A. Waldron, Quantum Attractor Flows, JHEP 09 (2007) 056 [arXiv: 0707.0267] [InSPIRE].

[42] T. Hakobyan and A. Nersessian, Lobachevsky geometry of (super)conformal mechanics, Phys. Lett. A 373 (2009) 1001 [arXiv:0803.1293] [InSPIRE].

[43] J. Maldacena, D. Martelli and Y. Tachikawa, Comments on string theory backgrounds with non-relativistic conformal symmetry, JHEP 10 (2008) 072 [arXiv:0807.1100] [INSPIRE].

[44] F. Correa, V. Jakubsky and M.S. Plyushchay, Aharonov-Bohm effect on $A d S_{2}$ and nonlinear supersymmetry of reflectionless Poschl-Teller system, Annals Phys. 324 (2009) 1078 [arXiv:0809.2854] [INSPIRE].

[45] P.D. Alvarez, J.L. Cortes, P.A. Horvathy and M.S. Plyushchay, Super-extended noncommutative Landau problem and conformal symmetry, JHEP 03 (2009) 034 [arXiv:0901.1021] [INSPIRE].

[46] A. Bagchi and R. Gopakumar, Galilean Conformal Algebras and AdS/CFT, JHEP 07 (2009) 037 [arXiv: 0902.1385] [INSPIRE].

[47] F. Correa, H. Falomir, V. Jakubsky and M.S. Plyushchay, Hidden superconformal symmetry of spinless Aharonov-Bohm system, J. Phys. A 43 (2010) 075202 [arXiv:0906.4055] [INSPIRE].

[48] T. Hakobyan, S. Krivonos, O. Lechtenfeld and A. Nersessian, Hidden symmetries of integrable conformal mechanical systems, Phys. Lett. A 374 (2010) 801 [arXiv:0908.3290] [INSPIRE].

[49] C. Chamon, R. Jackiw, S.-Y. Pi and L. Santos, Conformal quantum mechanics as the $C F T_{1}$ dual to $A d S_{2}$, Phys. Lett. B 701 (2011) 503 [arXiv:1106.0726] [INSPIRE].

[50] Z. Kuznetsova and F. Toppan, D-module Representations of $N=2,4,8$ Superconformal Algebras and Their Superconformal Mechanics, J. Math. Phys. 53 (2012) 043513 [arXiv:1112.0995] [INSPIRE].

[51] S. Fedoruk, E. Ivanov and O. Lechtenfeld, Superconformal Mechanics, J. Phys. A 45 (2012) 173001 [arXiv: 1112.1947] [INSPIRE].

[52] K. Andrzejewski, J. Gonera and P. Maślanka, Nonrelativistic conformal groups and their dynamical realizations, Phys. Rev. D 86 (2012) 065009 [arXiv:1204.5950] [InSPIRE].

[53] J. Gonera, Conformal mechanics, Annals Phys. 335 (2013) 61 [arXiv:1211.4403] [INSPIRE].

[54] J. Molina-Vilaplana and G. Sierra, An xp model on AdS $S_{2}$ spacetime, Nucl. Phys. B 877 (2013) 107 [arXiv: 1212.2436] [INSPIRE].

[55] M.S. Plyushchay and A. Wipf, Particle in a self-dual dyon background: hidden free nature and exotic superconformal symmetry, Phys. Rev. D 89 (2014) 045017 [arXiv:1311.2195] [INSPIRE].

[56] S.J. Brodsky, G.F. de Teramond, H.G. Dosch and J. Erlich, Light-Front Holographic QCD and Emerging Confinement, Phys. Rept. 584 (2015) 1 [arXiv:1407.8131] [INSPIRE].

[57] K. Andrzejewski, J. Gonera, P. Kosiński and P. Maślanka, On dynamical realizations of l-conformal Galilei groups, Nucl. Phys. B 876 (2013) 309 [arXiv: 1305.6805] [INSPIRE]. 
[58] M. Masuku and J.P. Rodrigues, De Alfaro, Fubini and Furlan from multi Matrix Systems, JHEP 12 (2015) 175 [arXiv:1509.06719] [INSPIRE].

[59] I. Masterov, Remark on higher-derivative mechanics with l-conformal Galilei symmetry, J. Math. Phys. 57 (2016) 092901 [arXiv: 1607.02693] [INSPIRE].

[60] K. Andrzejewski, Quantum conformal mechanics emerging from unitary representations of $\mathrm{SL}(2, \mathbb{R})$, Annals Phys. 367 (2016) 227 [arXiv: 1506. 05596] [INSPIRE].

[61] R. Bonezzi, O. Corradini, E. Latini and A. Waldron, Quantum Mechanics and Hidden Superconformal Symmetry, Phys. Rev. D 96 (2017) 126005 [arXiv:1709.10135] [INSPIRE].

[62] J. Mateos Guilarte and M.S. Plyushchay, Perfectly invisible $\mathcal{P} \mathcal{T}$-symmetric zero-gap systems, conformal field theoretical kinks and exotic nonlinear supersymmetry, JHEP 12 (2017) 061 [arXiv:1710.00356] [INSPIRE].

[63] L. Inzunza and M.S. Plyushchay, Hidden superconformal symmetry: Where does it come from?, Phys. Rev. D 97 (2018) 045002 [arXiv:1711.00616] [InSPIRE].

[64] H. Airault, H.P. McKean and J. Moser, Rational and elliptic solutions of the Korteweg-de Vries equation and a related many-body problem, Commun. Pure Appl. Math. 30 (1977) 95.

[65] M. Adler and J. Moser, On a Class of Polynomials Connected with the Korteweg-De Vries Equation, Commun. Math. Phys. 61 (1978) 1 [InSPIRE].

[66] A. Gorsky and N. Nekrasov, Hamiltonian systems of Calogero type and two-dimensional Yang-Mills theory, Nucl. Phys. B 414 (1994) 213 [hep-th/9304047] [INSPIRE].

[67] J.J. Duistermaat and F.A. Grünbaum, Differential equations in the spectral parameter, Commun. Math. Phys. 103 (1986) 177.

[68] A.P. Veselov, Huygens' principle and integrability, Prog. Math. 169 (1998) 259.

[69] S.P. Novikov, S.V. Manakov, L.P. Pitaevskii and V.E. Zakharov, Theory of Solitons, Plenum, New York (1984).

[70] I.M. Krichever, Baker-Akhiezer functions and integrable systems, in Integrability. The Seiberg-Witten and Whitham Equations, H.W. Braden and I.M. Krichever eds., Gordon and Breach Science Publishers, Amsterdam (2000).

[71] E.D. Belokolos, A.I. Bobenko, V.Z. Enol'skii, A.R. Its and V.B. Matveev, Algebro-Geometric Approach to Nonlinear Integrable Equations, Springer, Berlin (1994).

[72] F. Correa and M.S. Plyushchay, Hidden supersymmetry in quantum bosonic systems, Annals Phys. 322 (2007) 2493 [hep-th/0605104] [INSPIRE].

[73] F. Correa, L.-M. Nieto and M.S. Plyushchay, Hidden nonlinear supersymmetry of finite-gap Lame equation, Phys. Lett. B 644 (2007) 94 [hep-th/0608096] [INSPIRE].

[74] J.L. Burchnall and T.W. Chaundy, Commutative ordinary differential operators, Proc. Lond. Math. Soc. s2-21 (1923) 420 [Proc. Roy. Soc. Lond. A 118 (1928) 557].

[75] E.L. Ince, Ordinary differential equations, Dover (1956).

[76] F. Correa, V. Jakubsky, L.-M. Nieto and M.S. Plyushchay, Self-isospectrality, special supersymmetry and their effect on the band structure, Phys. Rev. Lett. 101 (2008) 030403 [arXiv:0801.1671] [INSPIRE].

[77] A. Arancibia, J. Mateos Guilarte and M.S. Plyushchay, Effect of scalings and translations on the supersymmetric quantum mechanical structure of soliton systems, Phys. Rev. D 87 (2013) 045009 [arXiv:1210.3666] [INSPIRE]. 
[78] C. Leiva and M.S. Plyushchay, Superconformal mechanics and nonlinear supersymmetry, JHEP 10 (2003) 069 [hep-th/0304257] [INSPIRE].

[79] F. Correa, M.A. del Olmo and M.S. Plyushchay, On Hidden broken nonlinear superconformal symmetry of conformal mechanics and nature of double nonlinear superconformal symmetry, Phys. Lett. B 628 (2005) 157 [hep-th/0508223] [INSPIRE].

[80] C.M. Bender, Making sense of non-Hermitian Hamiltonians, Rept. Prog. Phys. 70 (2007) 947 [hep-th/0703096] [INSPIRE].

[81] A. Mostafazadeh, Pseudo-Hermitian Representation of Quantum Mechanics, Int. J. Geom. Meth. Mod. Phys. 7 (2010) 1191 [arXiv:0810.5643] [INSPIRE].

[82] E.P. Wigner, Normal form of antiunitary operators, J. Math. Phys. 1 (1960) 409.

[83] A. Arancibia, F. Correa, V. Jakubský, J. Mateos Guilarte and M.S. Plyushchay, Soliton defects in one-gap periodic system and exotic supersymmetry, Phys. Rev. D 90 (2014) 125041 [arXiv: 1410.3565] [INSPIRE].

[84] A. Arancibia and M.S. Plyushchay, Chiral asymmetry in propagation of soliton defects in crystalline backgrounds, Phys. Rev. D 92 (2015) 105009 [arXiv:1507.07060] [INSPIRE].

[85] C.M. Bender and S. Boettcher, Real spectra in nonHermitian Hamiltonians having PT symmetry, Phys. Rev. Lett. 80 (1998) 5243 [physics/9712001] [INSPIRE].

[86] P. Dorey, C. Dunning and R. Tateo, Spectral equivalences, Bethe Ansatz equations and reality properties in $\mathcal{P} \mathcal{T}$-symmetric quantum mechanics, J. Phys. A 34 (2001) 5679 [hep-th/0103051] [INSPIRE].

[87] P. Dorey, C. Dunning and R. Tateo, Supersymmetry and the spontaneous breakdown of $\mathcal{P} \mathcal{T}$ symmetry, J. Phys. A 34 (2001) L391 [hep-th/0104119] [INSPIRE].

[88] M. Znojil, $\mathcal{P} \mathcal{T}$-symmetric harmonic oscillators, Phys. Lett. A 259 (1999) 220 [quant-ph/9905020] [INSPIRE].

[89] F. Correa and A. Fring, Regularized degenerate multi-solitons, JHEP 09 (2016) 008 [arXiv: 1605.06371] [INSPIRE].

[90] A. Fring and M. Znojil, $\mathcal{P} \mathcal{T}$-symmetric deformations of Calogero models, J. Phys. A $4 \mathbf{1}$ (2008) 194010 [arXiv:0802.0624] [INSPIRE].

[91] F. Correa and O. Lechtenfeld, $\mathcal{P} \mathcal{T}$ deformation of angular Calogero models, JHEP 11 (2017) 122 [arXiv: 1705.05425] [INSPIRE].

[92] R. El-Ganainy, K.G. Makris, M. Khajavikhan, Z.H. Musslimani, S. Rotter and D.N. Christodoulides, Non-Hermitian physics and PT symmetry, Nat. Phys. 14 (2018) 11.

[93] J.F. Cariñena and M.S. Plyushchay, ABC of ladder operators for rationally extended quantum harmonic oscillator systems, J. Phys. A 50 (2017) 275202 [arXiv:1701.08657] [INSPIRE].

[94] J.F. Cariñena, L. Inzunza and M.S. Plyushchay, Rational deformations of conformal mechanics, Phys. Rev. D 98 (2018) 026017 [arXiv:1707.07357] [INSPIRE].

[95] A. Arancibia and M.S. Plyushchay, Extended supersymmetry of the self-isospectral crystalline and soliton chains, Phys. Rev. D 85 (2012) 045018 [arXiv:1111.0600] [INSPIRE].

[96] S.P. Novikov, The periodic problem for the Korteweg-de Vries equation, Funct. Anal. Appl. 8 (1974) 236. 
[97] L. Inzunza and M.S. Plyushchay, Hidden symmetries of rationally deformed superconformal mechanics, Phys. Rev. D 99 (2019) 025001 [arXiv:1809.08527] [InSPIRE].

[98] F. Correa, O. Lechtenfeld and M. Plyushchay, Nonlinear supersymmetry in the quantum Calogero model, JHEP 04 (2014) 151 [arXiv: 1312.5749] [INSPIRE].

[99] S. Wojciechowski, Superintegrability of the Calogero-Moser system, Phys. Lett. A 95 (1983) 279.

[100] V.B. Kuznetsov, Hidden symmetry of the quantum Calogero-Moser system, Phys. Lett. A 218 (1996) 212 [solv-int/9509001] [INSPIRE].

[101] C. Gonera, A note on superintegrability of the quantum Calogero model, Phys. Lett. A 237 (1998) 365.

[102] M.F. Rañada, Superintegrability of the Calogero-Moser system: Constants of motion, master symmetries, and time-dependent symmetries, J. Math. Phys. 40 (1999) 236. 